\title{
Planetary nebula IC 5148 and its ionized halo*
}

\author{
D. Barría ${ }^{1}$, S. Kimeswenger ${ }^{1,2}$, W. Kausch ${ }^{2}$, and D. S. Goldman ${ }^{3}$ \\ ${ }^{1}$ Instituto de Astronomía, Universidad Católica del Norte, Av. Angamos 0610, Antofagasta, Chile \\ e-mail: daniela.barria@ucn.cl \\ 2 Institut für Astro- und Teilchenphysik, Leopold-Franzens Universität Innsbruck, Technikerstr. 25, 6020 Innsbruck, Austria \\ ${ }^{3}$ Astrodon Imaging, 407 Tyrolean Court. Roseville, California, 95661, USA
}

Received 29 July 2018 / Accepted 2 October 2018

\begin{abstract}
Context. Many round or roundish planetary nebulae $(\mathrm{PNe})$ show multiple shells and halo structures during their evolutionary stage near the maximum temperature of their central star. Controversial debate is ongoing if these structures are recombination halos, as suggested by hydrodynamic modeling efforts, or ionized material. Recently, we discovered a halo with somewhat unusual structures around the sparsely studied PN IC 5148 and present for the first time spectroscopy going out to the halo of such a PN.

Aims. We investigate the spatial distribution of material and its ionization state from the center of the nebula up to the very outskirts of the halo.

Methods. We obtained long-slit low resolution spectroscopy (FORS2 at VLT) of the nebula in two position angles, which we used to investigate the nebular structure and its halo in the optical range from 450 to $880 \mathrm{~nm}$. In addition we used medium resolution spectra taken with X-shooter at VLT ranging from $320 \mathrm{~nm}$ to $2.4 \mu \mathrm{m}$ to derive atmospheric parameters for the central star. We obtained the distance and position in the Galaxy from various methods combined with Gaia DR2 data. We also applied Cloudy models to the nebula in order to derive physical parameters of the various regions.

Results. We obtained spatially resolved structures and detailed descriptions of the outrunning shock front and a set of unusual halo structures denoted to further shock. The halo structures appears clearly as hot ionized material. Furthermore, we derived a reliable photometric value for the central star at a Gaia distance of $D=1.3 \mathrm{kpc}$. Considering the large distance $z=1.0 \mathrm{kpc}$ from the galactic plane together to its non-circular motion in the galaxy and, a metallicity only slightly below that of typical disk PNe, most likely IC 5148 originates from a thick disk population star.
\end{abstract}

Key words. planetary nebulae: general - planetary nebulae: individual: IC 5148 - stars: AGB and post-AGB

\section{Introduction}

Although the interacting wind model for the formation of planetary nebulae (PNe) has been accepted for a long time (Kwok et al. 1978; Kwok 1982, 2002) and morphological sequences were drawn in detail (Balick 1987), the exact mechanism is not yet clearly understood up to now. However, significant progress have been made in the past years. Several numerical simulations starting with Okorokov et al. (1985) followed by Frank \& Mellema (1994); Mellema \& Frank (1995), and by the Schönberner group (e.g. Perinotto et al. 1998, 2004; Steffen et al. 1998; Corradi et al. 2000; Schönberner et al. 2014), have been carried out. The latter performed a one-dimensional hydrodynamic simulation based on the evolutionary tracks and the simulations of the circumstellar shell around asymptotic giant branch (AGB) stars. The results of their simulation showed that a shock-bounded ionized main PN shell, moving supersonically into the AGB-material, compresses the inner parts of the matter into a dense but thin shell. The unaffected AGB-material becomes ionized as well and forms a rapidly expanding halo. Because of the drop in luminosity of the central star of the PN (CSPN) toward the white dwarf regime, the outer part of the PN shell recombines quickly forming a second inner halo. The disadvantage of this simulation is that it just considers one dimension. Thus, more complex interactions of the winds such as

\footnotetext{
^ Based on observations made with ESO Telescopes at the La Silla Paranal Observatory under program ID 098.D-0332
}

Rayleigh-Taylor instabilities, which quickly build a clumpy environment as we see in HST images, are not taken into account. For this reason, the filling factor $(\epsilon)$ has usually been set to unity. Moreover, comparing these models with observations of older evolved nebulae suffers from interaction to the environment and the interstellar matter (ISM). Thus, the study of nebulae with higher galactic latitudes is of special interest. However, there might be an influence on other properties changing the wind physics, mainly due to the lower metallicity. Photoionizing radiative transfer codes such as the steadily evolving Cloudy ${ }^{1}$ (Ferland et al. 1998, 2013, 2017; Ferland 2000) are often therefore used to characterize such nebulae and to identify their membership to the stellar populations (Emprechtinger et al. 2004, 2005; Öttl et al. 2014).

The PN IC 5148 (PN G002.7-52.4) is a nebula which is to date not well investigated in detail. First listed in the Second Index Catalogue of Nebulae and Clusters of Stars (Dreyer 1910) with two independent entries as numbers IC 5148 and IC 5150 discovered by Swift (1899) and Gale (1897) independently, it was finally discovered to be the same object by Hoffmeister (1961). Morphologically it is declared as a round nebula in all catalogs and Chu et al. (1987) classified it as a multiple shell planetary nebula (MSPN) due to a small step in the $\mathrm{H} \alpha$ image with a radius ratio of only 1:1.2 between the two structures. In addition, the intensity decrease was found smaller than that for typical

\footnotetext{
1 http://www.nublado.org
} 
MSPNe. A few years later the authors searched with a larger field systematically around many nebulae for extended emission features without detecting the very low surface brightness halo we investigate here (Hua et al. 1998). While earlier spectroscopic studies took only a small fraction of one or two spectral lines to obtain radial velocity and expansion of the nebula (e.g. Meatheringham et al. 1988), to our knowledge up to now the only, more extended spectroscopic analysis were performed by Kaler et al. (1990) and by Kingsburgh \& Barlow (1994) within two surveys of 75 and 80 southern PNe, respectively. Both studies used pre-CCD electronic spectral scanning devices, taking a very small aperture region of the nebula and detected only a hand full of lines. They end up coinciding in the result that the nebula has about galactic disk abundance or only slight underabundance despite its large galactic latitude $\left(b\right.$ approximately $\left.-52^{\circ}\right)$. Further, the survey spectra used in the PN catalog of Acker et al. (1992) gives intensities of only four lines. The inspection of the original data file of this survey, provided now at the Hong Kong/AAO/Strasbourg PN data base (thereafter $\mathrm{HASH}^{2}$; Parker et al. 2016; Bojičić et al. 2017), does not recover more usable lines above the noise level.

The CSPN of IC 5148 was first classified by Acker et al. (1982), and studied in detail by using the International Ultraviolet Explorer (IUE) by Kaler \& Feibelman (1985). They revealed that it is a very evolved hot central star. The IUE color index was not sufficient to derive an exact value of the temperature, but they give values based on their three methods from approximately $100-220 \mathrm{kK}$. As later stated by Patriarchi \& Perinotto (1991b,a), using the same data, no strong wind features were found. Moreover, a later investigation shows that this is common for CSPN of nebulae which have passed the knee in the Hertzsprung-Russell diagram (HRD) toward the beginning white dwarf cooling track. The only published optical study of the CSPN is the classification of Mendez (1991), giving it the type of a $\mathrm{hgO}(\mathrm{H})$ star (a hydrogen rich high gravity star with very broad Balmer absorptions).

Previous distance estimations obtained from so-called statistical distance scales span from $0.53 \mathrm{kpc}$ using the CSPN evolution (Phillips 2005), $1.06 \mathrm{kpc}$ using the ionized mass versus surface brightness relation (Cahn et al. 1992; Stanghellini et al. 2008), $1.1 \mathrm{kpc}$ using the IRAS dust temperature (Tajitsu \& Tamura 1998), $1.37 \mathrm{kpc}$ by using the $\mathrm{H} \alpha$ surface brightnessradius relation (Frew et al. 2016), $1.8 \mathrm{kpc}$ using a non-linear calibration of the radio brightness vs. diameter (using Buckley \& Schneider 1995, calculated by us), $2.1 \mathrm{kpc}$ using the radio temperature vs. radius relation (using van de Steene \& Zijlstra 1994, calculated by us) and even $3.59 \mathrm{kpc}$ using the mass-radius distance (Zhang 1995).

In this paper we present the recent discovery of extended emission features around the hitherto known nebula IC 5148 and a detailed medium-high resolution spectroscopic study of its central star with the ESO VLT X-shooter instrument. We also performed a detailed spectroscopic study with long-slit spectroscopy from ESO FORS2 in various directions of the nebula and the newly discovered halo. The latter is, to our knowledge, one of the first and deepest yet found of its type in a halo. Finally, using the now available distance estimation from the second data release DR2 of Gaia (Gaia Collaboration 2018) and the derived parameters from our CSPN spectroscopy, we modeled the main nebula by using the photoionizing radiative transfer code Cloudy (Ferland et al. 2017).

\footnotetext{
2 http://hashpn.space/
}

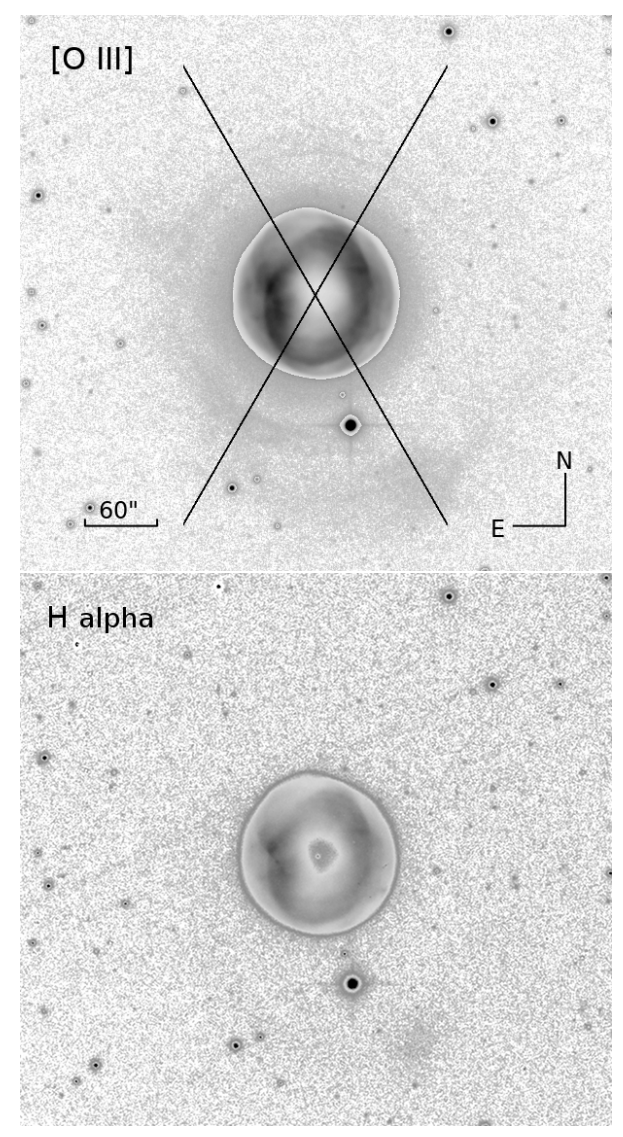

Fig. 1. Co-added direct images in [O III] (top panel) and $\mathrm{H} \alpha$ (bottom panel). The slit positions of the spectroscopy are indicated in the top panel. While in the inner part, a linear intensity scale covering the whole range from zero to peak flux of the nebula was used, for regions with flux below $5 \%$ a steep asinh between 0.01 and $1 \%$ of the peak of the linear part was used to enhance the by orders of magnitude fainter structures.

\section{Data}

The narrow band imaging was derived at the CDK20 Planewave telescope of ITelescope.net in Siding springs (31 $16^{\prime} 24^{\prime \prime}$ south, $149^{\circ} 03^{\prime} 52^{\prime \prime}$ east) during several nights in July 2013. An Apogee U16MD9 camera equipped with a Kodak 16803 CCD without focal reducer and without binning was used. A total exposure time of $11.0 \mathrm{~h}$ with the [O III] filter by co-adding 22 frames and $4.5 \mathrm{~h}$ with the $\mathrm{H} \alpha$ filter by co-adding nine exposures were obtained. The resulting images, shown in Fig. 1, were used to identify the features of interest and the position angles for the follow up investigations. To obtain a correct line ratio [O III] $/ \mathrm{H} \alpha$ image, the resulting frames were differentially calibrated by means of the long-slit spectroscopy derived at ESO. The astrometry was determined by 40 surrounding stars of the Gaia DR2 catalog (Gaia Collaboration 2018) resulting in a plate scale of 0 '.5447 per pixel. Stars near the nebula on the co-added images have a full width at half maximum (FWHM) of 1.'82 and 1.'96 in $\mathrm{H} \alpha$ and [O III], respectively.

Long-slit spectra were obtained with the FORS2 spectrograph (Appenzeller et al. 1998) mounted on the Cassegrain focus of ESO VLT UT1 (Antu) in the nights October 5, 2016 from 3:15 to 4:03 UT; October 6, 2016 from 0:15 to 0:30 UT and, October 10, 2016 from 1:17 to 1:45 UT in service mode as filler program with minor sky quality. In total, seven spectra were collected, 
of which, three have a position angle $\mathrm{PA}=30^{\circ}$ and four have a position angle $\mathrm{PA}=150^{\circ}$ (from $\mathrm{N}$ over $\mathrm{E}$ ) on the sky through the center of the PN. The slit positions were selected to cover the features in the wide halo of the PN. We used the long-slit mode of FORS2 since this enabled us to cover the entire halo using the full slit length of 6.8 with the standard collimator (slit width $=0 \prime^{\prime} 7$ ). All spectra were taken with 14 min. exposure time. We used GRISM 300V and the GG435 order separation filter, covering a wavelength range from 455 to $889 \mathrm{~nm}$. The MIT/LL CCD mosaic and the standard focal reducer collimator result in a 0.2518 pixel $^{-1}$ spatial scale. This setup leads to a final wavelength dispersion of $0.33 \mathrm{~nm}$ pixel $^{-1}$. The night sky lines were measured with a resolution $R=\lambda / \Delta \lambda$ of 200 and $R=360$ at the blue and the red ends of the spectrum, respectively. We derived a FWHM of the stellar sources along the slit of about 1 ". 17 at the blue end and 0.'95 at the red part of the spectrum. This corresponds well to the reported DIMM seeing of the ESO meteo monitor of 1 .' 1 at $500 \mathrm{~nm}$. The data were reduced incorporating the standard calibration mode using the ESO FORS2 pipeline v5.3.11 3 (Freudling et al. 2013). The resulting flux calibration was compared with the expected continuum flux of the CSPN, finding a scatter of less than $5 \%$ between the individual observations obtained at clear, but non-photometric sky conditions. Two spectra at position angle $30^{\circ}$ were taken at slightly minor sky conditions with some cirrus clouds on October 5, 2016. These were scaled with a constant wavelength independent factor using the central star observations. We used the software package molecfit (Smette et al. 2015; Kausch et al. 2015) to create a model of the telluric absorption lines by applying it on the high signal-to-noise ratio $(\mathrm{S} / \mathrm{N})$ central star spectrum. As the PN and its extended halo covered the whole slit length, we used the software skycorr (Noll et al. 2014) to correct for the sky emission lines. This approach also enabled us to derive a secondary correction of the wavelength calibration. We averaged the spectra for each sky direction to achieve the final spectra of the faint sources used for this study. Finally, a correction by photometric values of $20 \%$ was applied (see Sect. 3 ).

The medium-high resolution echelle spectra were taken with the X-shooter instrument (Vernet et al. 2011) mounted at the ESO VLT UT2 (Kueyen) in the night of October 5, 2016 from 0:23 to 0:57 UT at slightly degraded weather conditions as filler program in service mode. This instrument covers the entire wavelength range from 0.3 to $2.5 \mu \mathrm{m}$ in three spectral arms (UVB arm from the ultraviolet to the $B$ band with an E2V CCD44-82; VIS arm in the visual regime with a MIT/LL CCD; NIR arm in the nearinfrared regime with an Hawaii2RG chip), at medium resolution simultaneously. While the NIR and UVB frames had an exposure time of $125 \mathrm{~s}$ each, the VIS arm exposures were $213 \mathrm{~s}$ each (all $0.8^{\prime \prime}$ slit). A total of 29 frames were obtained and co-added during data reduction with the standard ESO X-shooter pipeline v2. $8^{4}$ (Modigliani et al. 2010). The spectra were normalized to continuum to derive line profiles for the spectral analysis. Thus, the slightly degraded weather conditions did not harm. We did not apply a telluric absorption correction since all lines of interest were in undisturbed regions. The $\mathrm{S} / \mathrm{N}$ was about 45 in the UVB and 25 in the VIS arm. That was lower than expected from exposure time estimators and the DSS magnitude of the central star. It could be caused by a combination of cirrus clouds and the $1.6^{\prime \prime}$ seeing at that time. The $\mathrm{S} / \mathrm{N}$ of the NIR part of the spectrum

\footnotetext{
3 http://www.eso.org/sci/software/pipelines/fors/ fors-pipe-recipes.html

4 https://www.eso.org/sci/software/pipelines/xshooter/ xshooter-pipe-recipes.html
}

was insufficient to derive any parameters and so was not used further.

\section{Analysis}

\subsection{The central star}

The X-shooter spectrum was normalized to unity flux and searched for absorption lines. The only one appearing in the VIS arm spectrum was the $\mathrm{H} \alpha$ line. Nevertheless, it is heavily contaminated by the emission of the Hydrogen line by the nebula and by the two strong [N II] lines. As shown below in the following section, these lines are asymmetric and doubled due to nebular expansion. Moreover, they vary significantly along the slit. Thus, a reliable removal was not possible and the $\mathrm{H} \alpha$ line was not used for the further analysis. The NIR arm has a too-low S/N value ( $<10$ in the $J$ band and $<5$ in the $H$ and $K$ bands) to derive a useful spectrum. Therefore, the analysis of the CSPN parameters is focused only on the UVB arm of the X-shooter instrument.

We overlaid in our analysis the high resolution non-LTE spectra for central stars of planetary nebulae of the Tübingen stellar atmosphere (thereafter TMAP, Rauch 2003) as offered at Rauch \& Reindl (2014) to the identified lines in the UVB arm spectra (Fig. 2). The clear appearance of the He II $468.6 \mathrm{~nm}$ line in absorption, not seen by Mendez (1991), and the He II $420.0+454.1 \mathrm{~nm}$ lines without major absorption, places the CSPN in their classification as $\mathrm{O}(\mathrm{H})$ and not as $\mathrm{hgO}(\mathrm{H})$. The number of lines and the $\mathrm{S} / \mathrm{N}$ does not allow for a sophisticated determination of the $\log (g)$ value. Thus, the evolution along a standard model by Miller Bertolami (2016) was assumed. As Napiwotzki (2001) showed, the result is not sensitive on the selection of $\log (g)$ for the optical flux analysis. A likelihood analysis of all lines, weighted by equivalent width of the line to consider the weakness of the lines like He II $454.1 \mathrm{~nm}, 420.0 \mathrm{~nm}$ or $\mathrm{H} \epsilon$, was used to derive a $T_{\text {eff }} \approx 140_{-13}^{+5} \mathrm{kK}(\log T=5.146)$ for the central star.

The calibrated FORS2 spectra of the CSPN were first compared individually and then combined to derive the final flux. The derived flux curve was folded with the filter curves of Bessell (1990) and multiplied with the zero point given in Bessell et al. $(1998)^{5}$. This results in $V=16.34$. The DENIS $I_{\mathrm{C}}=16.476 \pm 0.10$ lead with a typical color of such very hot white dwarfs $\left(V-I_{\mathrm{C}}\right) \approx-0.35 \pm 0$. 05 from Landolt \& Uomoto (2007) for a $V_{\text {DENIS }}=16.13 \pm 0.15$, but the target is very much near the survey limit. Kaler \& Feibelman (1985) derived magnitudes of $V(\mathrm{UV})=16^{\mathrm{m}} \cdot 16 \pm 0 .{ }^{\mathrm{m}} 17$ using the IUE spectra between 130 and $185 \mathrm{~nm}$ and assuming a $130000 \mathrm{~K}$ blackbody spectrum and zero extinction due to the high galactic latitude. The flux calibration allows us to directly estimate the interstellar foreground extinction to be very low. The Balmer decrement of the nebular lines in our FORS2 spectra (only $\mathrm{H} \alpha$ and $\mathrm{H} \beta$ are covered by these spectra) give us an $E(B-V)=0$. $031 \pm 0.010$. The central star continuum, using stellar models from the Tübingen Model Atmosphere Package TMAP (Rauch 2003) accessed via the German Astrophysical Virtual Observatory (GAVO) service TheoSSA (Rauch \& Ringat 2011), confirm the almost extinctionfree line of sight. The Galaxy Evolution Explorer (GALEX) data base give the similar value of $E(B-V)=0$. 021 based on the NUV flux of $m_{\mathrm{NUV}}=13.480 \pm 0$. 004. Using the GALEX FUV

\footnotetext{
5 In Bessell et al. (1998) Table A.2, the $z p\left(f_{\lambda}\right)$ and the $z p\left(f_{\nu}\right)$ are reversed in the formulas for the magnitudes - confirmed by author in private communication.
} 

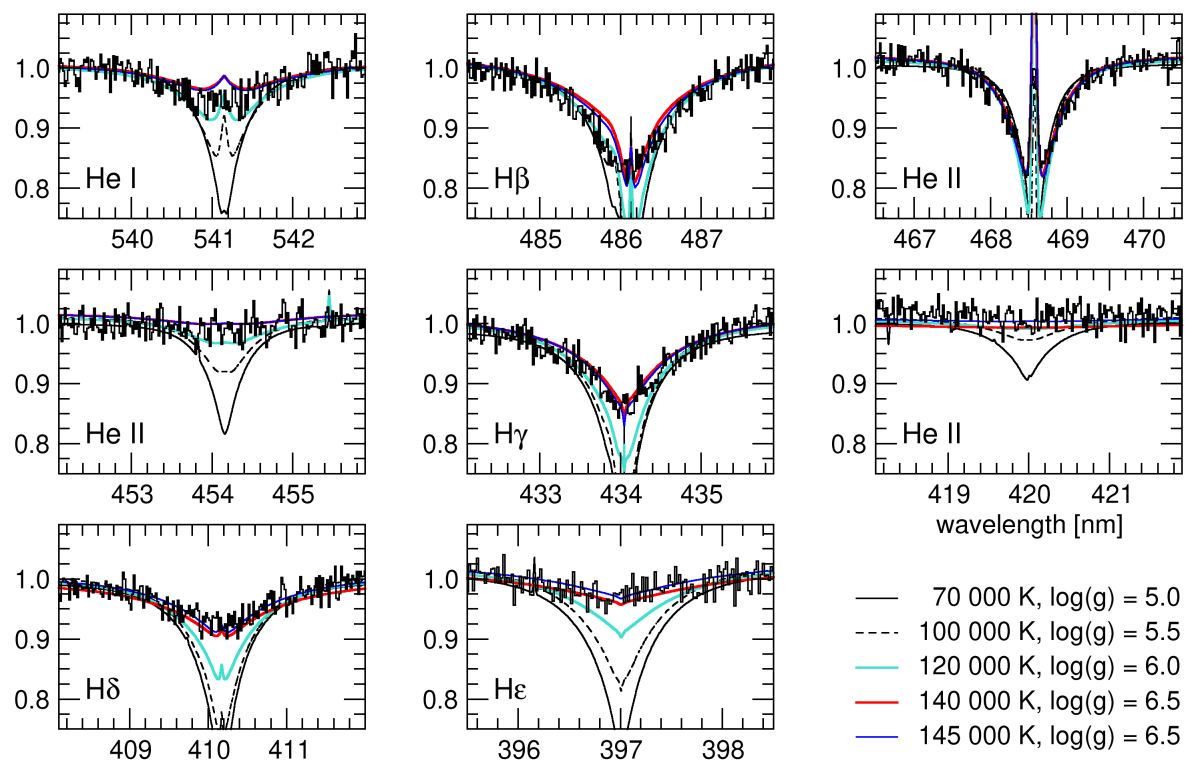

- $\quad 70000 \mathrm{~K}, \log (\mathrm{g})=5.0$ $100000 \mathrm{~K}, \log (\mathrm{g})=5.5$ $120000 \mathrm{~K}, \log (\mathrm{g})=6.0$ $140000 \mathrm{~K}, \log (\mathrm{g})=6.5$

$-145000 \mathrm{~K}, \log (\mathrm{g})=6.5$
Fig. 2. Spectral lines from the medium-high resolution spectroscopy of the UVB Arm of $\mathrm{X}$-shooter. Overlaid are the model grid spectra along the evolutionary track $\log (g)$ values of a CSPN according to Miller Bertolami (2016) from the high resolution stellar spectra grid from the German Astrophysics Virtual Observatory (GAVO) grid resources (Rauch \& Reindl 2014).

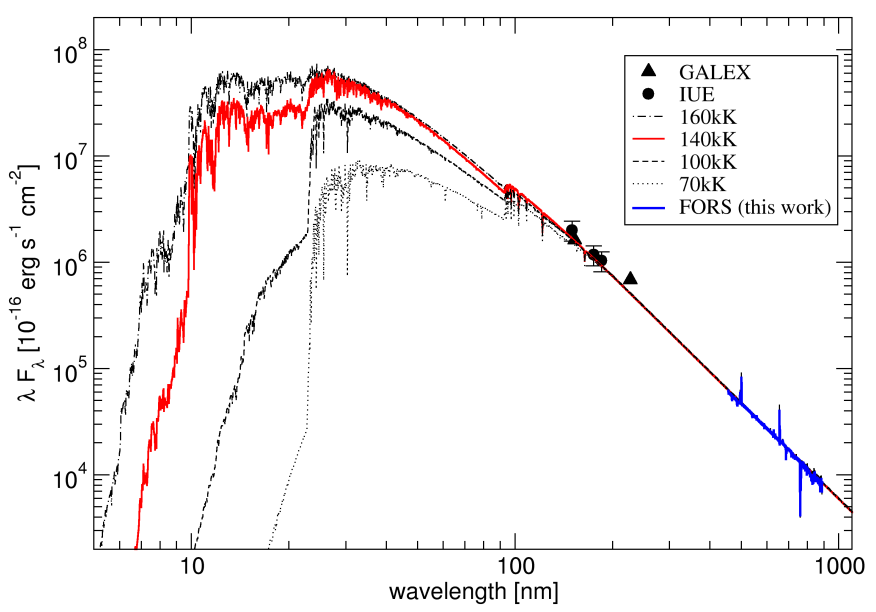

Fig. 3. UV photometry from IUE and GALEX with our calibrated FORS2 spectrum. The model spectral energy distributions from TMAP are overlaid, normalized to the FORS2 data.

data in the small 7 " aperture and applying that to the TMAP NLTE model together with the effective temperature derived by our spectroscopy with X-shooter and the interstellar extinction obtained from the Balmer lines, with the extrapolation as used in Kaler \& Feibelman (1985) for IUE data, we achieve a visual magnitude of 16.35 . The spectral energy distribution (SED) from 130 to $800 \mathrm{~nm}$ fits very well using a low extinction value of $E(B-V)=0$ m03 (see Fig. 3). The overlaid TMAP spectra also show how marginally the SED differ for these stars even at IUE and GALEX wavelengths, and thus why Kaler \& Feibelman (1985) were unable to derive a more accurate CSPN temperature. We further adopted this low value for the interstellar extinction. This value is in contradiction to the spectroscopy by Kaler et al. (1990) and Kingsburgh \& Barlow (1994), who found, using several lines of the Balmer decrement, values as high as $c(\mathrm{H} \beta)$ of 0.4 and 0.38 , respectively (corresponding to $E(B-V)=0.26$ ). However, using their published line intensities from $\mathrm{H} \alpha, \mathrm{H} \beta$, $\mathrm{H} \gamma$ and $\mathrm{H} \delta$ individually give the large scatter of values of $c$ from even slightly negative values of -0.03 up to 0.85 . An extinction value as high as $c=0.4$ does neither match with our spectra, nor with the IUE and GALEX photometry. Moreover, it is also unlikely with respect to the high galactic latitude of the target ${ }^{6}$. On the other hand, the $\mathrm{H} \alpha / \mathrm{H} \beta=1.7$ in Acker et al. (1992) is nonphysical. We thus take these values no further into account.

There are some issues with Gaia DR2 for the extraction of nebular contamination (see Kimeswenger \& Barría 2018). However, since the central star of IC 5148 appears isolated from its main nebula, we expect for a mostly reliable photometry from Gaia. We thus compared isolated blue compact white dwarfs in the lists of Landolt photometric standards (Landolt 1992) and spectrophotometric standards (Landolt \& Uomoto 2007) to derive color equations dedicated to this type of stars for the photometry into standard system (see Fig. 4). With this we derive a $V_{\text {Gaia }}=16.14 \pm 0.03$, using $G_{\text {Gaia }}=16$. 097 and Gaia color $(\mathrm{BP}-\mathrm{RP})=-0.635$. We finally adopted the latter value as reliable, and corrected the FORS2 fluxes by $20 \%$ to take into account the filler program minor sky conditions.

To test the result and to approve for the photometric flux calibration, the FORS2 spectrum was weighted with $\lambda^{4}$ (giving for the Raleigh Jeans tail of an exact blackbody a flat spectrum) and compared with the TMAP model downgraded to the FORS2 resolution (see Fig. 5). The deviation of the continuum is in most parts on the order of a few percent. The four stellar lines in the region, namely, $\mathrm{H} \alpha, \mathrm{H} \beta, \mathrm{He}$ I $540.6 \mathrm{~nm}$, and $\mathrm{He}$ II $468.5 \mathrm{~nm}$ (taking into account the slight error in the continuum calibration at the blue end) also fit perfectly in their depths. The latter together to the fact that the $\mathrm{He} \mathrm{I/He} \mathrm{II} \mathrm{ratio} \mathrm{is} \mathrm{very} \mathrm{sensitive} \mathrm{to} \mathrm{the}$ stellar temperature, independently confirms our X-shooter result on $T_{\text {eff }}$.

\subsection{Nebular expansion, systemic velocity and astrometry}

The X-shooter spectra have only a $9^{\prime \prime}$ long slit and thus do not cover the nebula as a whole. But its high resolution allows the determination of the line splitting along the line of sight in the center of the nebula, thus giving the direct value for the nebular expansion velocity (see Fig. 6). Moreover, the midpoint of the two lines gives a good value for the systemic velocity. Low resolution spectroscopy often suffer from the fact that the two unresolved lobes are not identical in intensity. Thus, for example,

\footnotetext{
6 https://ned.ipac.caltech.edu/?q=extinction_ calculator provided by the NED data base and based on Schlafly \& Finkbeiner (2011).
} 


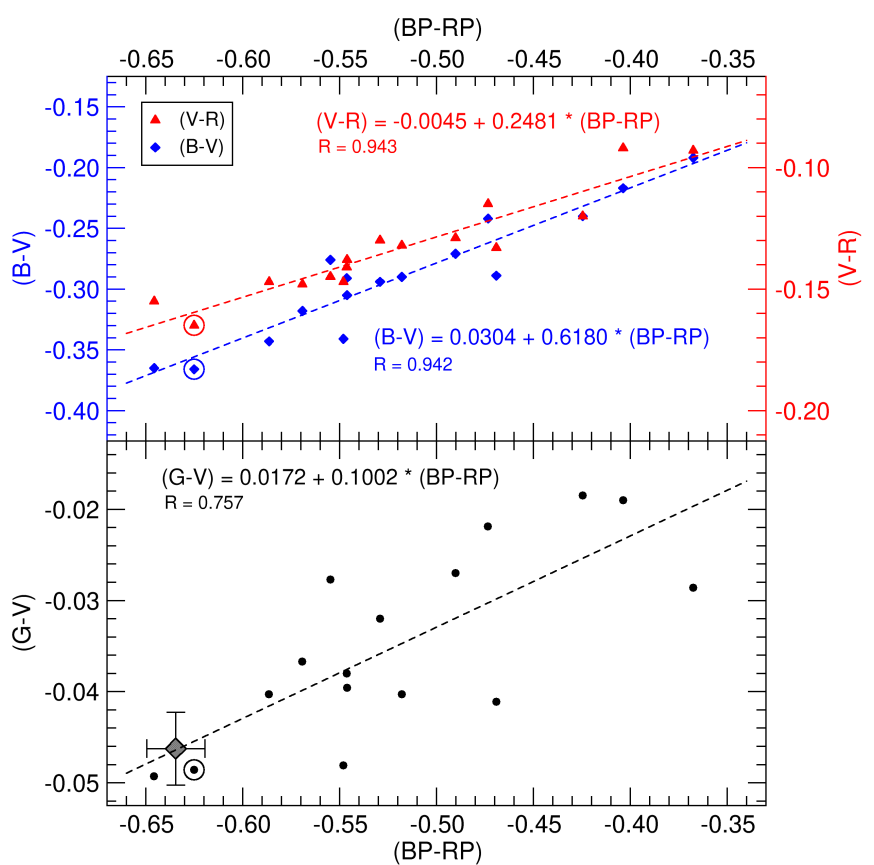

Fig. 4. Color equations of hot white dwarfs from the Landolt photometric standards (Landolt 1992) and from the spectrophotometric standards (Landolt \& Uomoto 2007) versus Gaia DR2. The target position used for calibrations is given in the lower panel. While all other stars are hot nearby compact white dwarfs without PNe, the encircled target is the central star of the Helix nebula.

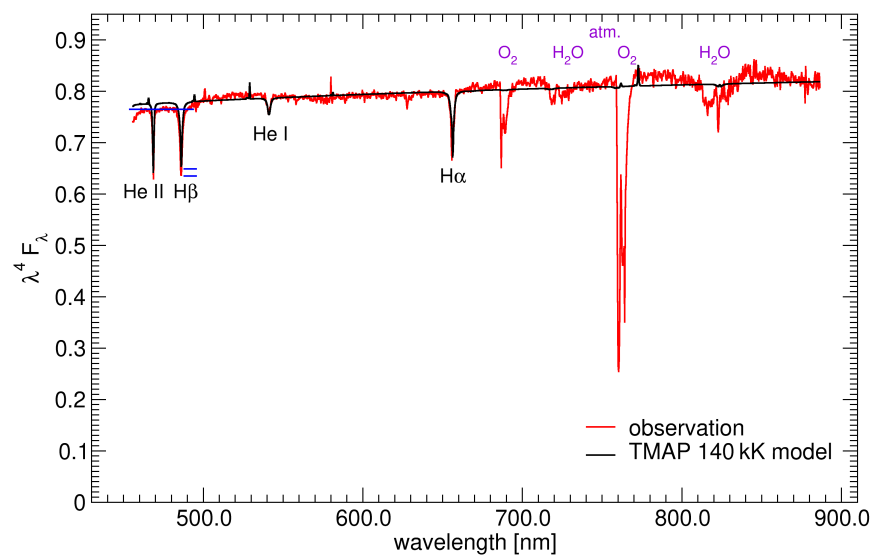

Fig. 5. $\lambda^{4}$ weighted FORS2 spectrum with the TMAP model. We intentionally did not remove the telluric $\mathrm{O}_{2}$ and $\mathrm{H}_{2} \mathrm{O}$ absorptions at the red end of the spectrum. The blue horizontal lines show the required correction of the line depths at the blue end due to the deviation to the continuum.

in the case of unresolved lines the result would be biased toward to red values. The measured expansions were independent of the element type within the error. This is in fact astonishing, as the high ionized species often give higher expansion velocities than the low ionized species (Weinberger 1989; Gesicki et al. 2014). We measured a peak to peak expansion velocity of $47.9 \pm 1.5 \mathrm{~km} \mathrm{~s}^{-1}$. This is in very good agreement with the value of $53.4 \mathrm{~km} \mathrm{~s}^{-1}$ given by Meatheringham et al. (1988) if taking into account the much lower spectral resolution of $11.5 \mathrm{~km} \mathrm{~s}^{-1}$ used by the authors. The nebula thus has a fairly large expansion compared to other roundish nebula in the catalogs (e.g. Weinberger 1989, and citations therein). The center between the

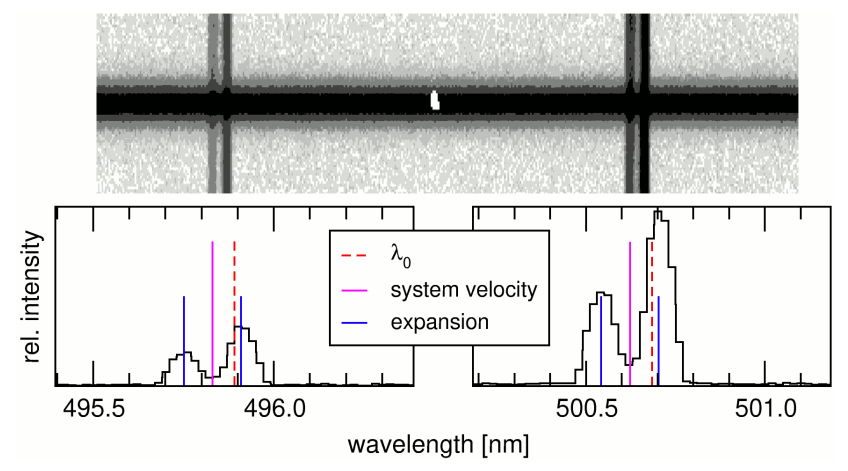

Fig. 6. Expansion of the PN and system velocity in the Galaxy from $\mathrm{X}$-shooter spectra after correction for LSRK.

two well separated peaks was used as system velocity. The measured system blueshift was corrected for the local standard of rest (LSRK) using the $\mathrm{IRAF}^{7}$ procedure rvcorrect. This result in $R V_{\mathrm{LSRK}}=-36.7 \pm 1.7 \mathrm{~km} \mathrm{~s}^{-1}$. The systematic difference from the hitherto published value of $-23 \mathrm{~km} \mathrm{~s}^{-1}$ (Meatheringham et al. 1988) most likely originates from the asymmetry of intensities of the redshifted and of the blueshifted part of the shell. This moves the center toward the brighter peak in the case of low resolution spectroscopy.

The astrometry was derived by 40 surrounding stars from the Gaia DR2 catalog (Gaia Collaboration 2018) which resulted in a rms of about 105 mas. We found only one old plate, which was scanned with high resolution from the ESO red survey plate scanned at MAMA and provided via CDS Aladin. It dates from September 5 1985. This was calibrated in the same homogeneous way with the same Gaia stars. The rms of this calibration was 80 mas. The resulting positions using our astrometric solution and that of Gaia (Lindegren et al. 2018) are given by

Gaia DR2: Epoch 2015.5, Equinox J2000.0

$$
\begin{array}{rll}
\alpha & =21^{\mathrm{h}} 59^{\mathrm{m}} 35^{\mathrm{s}} .095392053 & \pm 0.000000001 \\
\mu_{\alpha} & =-0.00652 \operatorname{arcsec}_{\mathrm{yr}}{ }^{\mathrm{s}} & \\
\delta & =-39^{\circ} 23^{\prime} 08^{\prime \prime} 35833212 & \pm 0^{\prime} .00000002 \\
\mu_{\delta} & =-0^{\prime} .00779 \operatorname{arcsec~yr}^{-1} &
\end{array}
$$$$
\text { CDK20: Epoch 2013.52, Equinox J2000.0 }
$$$$
\alpha=21^{\mathrm{h}} 59^{\mathrm{m}} 35^{\mathrm{s}} .1066 \quad \pm 0.0080
$$$$
\delta=-39^{\circ} 23^{\prime} 08^{\prime \prime} .439 \quad \pm 0{ }^{\prime} 12
$$$$
\text { ESO R: Epoch 1985.68.5, Equinox J2000.0 }
$$$$
\begin{array}{rll}
\alpha & =21^{\mathrm{h}} 59^{\mathrm{m}} 35^{\mathrm{s}} .1084 & \pm 0.0053 \\
\delta & =-39^{\circ} 23^{\prime} 08^{\prime \prime} \cdot 714 & \pm 0 !^{\prime} 08
\end{array}
$$

While the ESO R plate corresponds perfectly using the Gaia proper motion, the stacked CDK20 images have a offset slightly bigger than the error bars, most likely having its origin in a remaining distortion correction on the stacked images. But as it is still far below the FWHM caused by the seeing, we are still able to trust the division of images to derive line ratios. Using the Gaia DR2 distance of $1.3 \mathrm{kpc}$ (see Sect. 3.3) we derive a proper motion velocity in the plane of sight of $62 \pm 5 \mathrm{~km} \mathrm{~s}^{-1}$ toward southeast.

\subsection{Distance, luminosity, and position in the Galaxy}

The most commonly used statistical distance scales are that one of Cahn et al. (1992) and Stanghellini et al. (2008) giving a distance to IC 5148 of $1.06 \mathrm{kpc}$. Using the same radio data, but

7 Image Reduction and Analysis Facility available at
http://iraf.noao.edu/




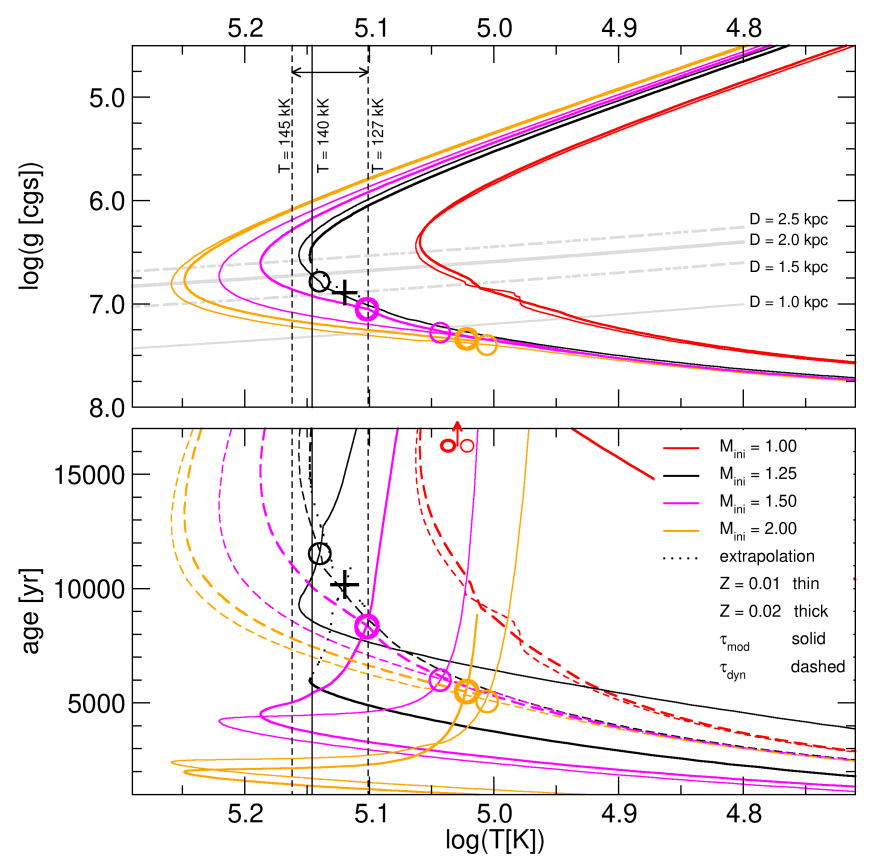

Fig. 7. Upper panel: evolutionary tracks of Miller Bertolami (2016) as $\log (g)$ vs. $\log (T)$ for initial masses of $1.00,1.25,1.5$ and $2.0 M_{\odot}$ (color coded) for metallicities of $Z=0.01$ and $Z=0.02$ (line thickness coded). The corresponding spectroscopic distances are indicated in light gray. The vertical dark gray lines indicate the range of temperatures as derived from our spectroscopy. Dotted lines mark extrapolation of the track, which ended earlier. Lower panel: computed model ages from the tracks above (solid lines) vs. the dynamical ages (dashed lines). The circles indicate the solutions by intersections of the model age with the dynamical age. The corresponding solutions found in the lower panel are marked in the upper panel as well. Crosses mark the estimated solution of the extrapolated track. The intersections of the $1.0 M_{\odot}$ (red) fall far outside the graph.

the radio brightness temperature method of van de Steene \& Zijlstra (1994) we obtain a distance of $2.1 \mathrm{kpc}$. More recently, Frew et al. (2016) established a method based on the optical $\mathrm{H} \alpha$ surface brightness giving $1.37 \mathrm{kpc}$. Using a recent calibration with the Gaia DR1 data for the statistical methods mentioned above by Stanghellini et al. (2017), we then get a distance of $1.88_{-0.48}^{+0.53} \mathrm{kpc}$. In a new investigation, containing a large sample of sources, Kimeswenger \& Barría (2018) showed for some issues concerning Gaia DR2 (Gaia Collaboration 2018; Luri et al. 2018) parallaxes for PNe with high surface brightness around the central stars. However, in this case the blue Gaia color (BP-RP) suggests for no contamination, and so we believe we are able to use the value provided by Gaia. Thus, we get a Gaia distance of $D_{\text {Gaia }}=1.28_{-0.13}^{+0.16} \mathrm{kpc}$. For the further analysis a final distance of $1.3 \mathrm{kpc}$ is applied.

The radial velocity, accurate proper motion and the Gaia distance allows us to derive the spatial movement vector. The target moves in galactic coordinates toward lower galactic longitude at $22 \mathrm{~km} \mathrm{~s}^{-1}$ and marginally increases its distance below the galactic plane with $-7 \mathrm{~km} \mathrm{~s}^{-1}$ while it moves to larger galactocentric distance with $+68 \mathrm{~km} \mathrm{~s}^{-1}$. Thus, while the peculiar fraction from normal galactic rotation and change in $z$ is small, it is significantly moving outwards in the Galaxy.

Using our spectra and photometry of the central star we are also able to derive a spectroscopic distance (Napiwotzki 1999, 2001) by means of the dereddened apparent magnitude, the mass
Table 1. Derived input parameters for the CSPN.

\begin{tabular}{lll}
\hline \hline Parameter & Symbol & Value \\
\hline Effective temperature & $T_{\text {eff }}$ & $130 \mathrm{kK}$ \\
& $\log \left(T_{\text {eff }}\right)$ & 5.114 \\
Initial mass & $M_{\text {ini }} / M_{\odot}$ & 1.50 \\
Final mass & $M_{\text {fin }} / M_{\odot}$ & 0.576 \\
Luminosity & $L / L_{\odot}$ & 320 \\
& $\log \left(L / L_{\odot}\right)$ & 2.51 \\
Surface gravity & $\log (g[\mathrm{cgs}])$ & 7.05 \\
Model age & $\tau_{\mathrm{mod}}$ & 8500 years \\
& & \\
Apparent brightness & $m_{\mathrm{V}}$ & 116.14 \\
Distance & $D$ & $1.3 \mathrm{kpc}$ \\
Galactic plane distance & $z$ & $-1.0 \mathrm{kpc}$ \\
\hline
\end{tabular}

of the central star and, the surface gravity $\log (g)$. With a temperature derived from the $\mathrm{X}$-shooter data we obtained a range of distances. We compared these to the recent evolutionary tracks (Fig. 7) by Miller Bertolami (2016). We derived with the evolutionary tracks, the spectroscopic model distances and with the expansion velocity, a dynamical age $\tau_{\text {dyn }}$ for each, according to

$\tau_{\text {dyn }}=\theta v_{\text {exp }} D_{\text {phot }}\left(T, M_{\text {fin }}, V_{\text {Gaia }}\right)$,

where $\theta=677^{\prime \prime} 2$ is the angular radius averaged over all directions, $v_{\exp }=47.9 \mathrm{kms}^{-1}$ the expansion velocity, and $D_{\text {phot }}$ is a distance as function of the effective temperature $T$ (formula 1 in Napiwotzki 2001) using the final mass $M_{\text {fin }}$ of the track and the photometry $V_{\text {Gaia }}$. This was then compared to the model age $\tau_{\text {mod }}$. Even if we take into account an uncertainty factor of two in the dynamical age, the intersection within the given temperature range derived for the CSPN fits only for the following tracks: initial mass $M_{\text {ini }}=1.25 M_{\odot}$, metallicity $Z=0.01$ giving a distance of $D=1.8 \mathrm{kpc}$ and, $M_{\text {ini }}=1.5 M_{\odot}$, metallicity $Z=0.02$ ending up in $D=1.3 \mathrm{kpc}$. Extrapolating the track for $M_{\text {ini }}=1.25 M_{\odot}$, metallicity $Z=0.02$ (which stopped due to a late helium flash in the calculation of the stellar model grid) we derive an intermediate position at $D=1.5 \mathrm{kpc}$. We obtain similar results within $20 \%$ using the tracks of Vassiliadis \& Wood (1994) and assuming an initial to final mass relation (Vassiliadis \& Wood 1993) to derive mass and $\log (g)$. The tracks of Blöcker (1995) do not cover such low mass stars. This intersection lowers slightly, but within the error by $0.8 \sigma$ the effective temperature of the star as derived above from the X-shooter spectra. Any other intersection of dynamical age with model age do not fit by more than a factor of three. Hence, these evolutionary tracks are in good agreement with the distances and temperatures previously derived from the nebular parameters and lead us to the input parameter set for the best fitting model with a initial mass of $M_{\text {ini }}=1.5 M_{\odot}$ and $Z=0.02$. The resulting parameters are listed in Table 1 .

\subsection{Line nebular analysis}

While the lines of $\mathrm{H} \alpha, \mathrm{H} \beta$, [O III] and $\mathrm{He}$ I follow well the same spatial behavior of the main nebula rim (Fig. 8, upper panel), there is a clear concentration of the low ionized species [N II] and [S II] toward the outer edge (Fig. 8, middle). Moreover, the central concentration of the high ionized species He II and [Ar IV] relative to the lower ionization lines of He I (Fig. 8, lower panel) is more pronounced. The four cuts given by the two slits from the central star outwards in radial distance show fairly the same shapes, hence the lines were averaged as radial profiles for the Cloudy modeling below. The line ratio [O III] $500.7 \mathrm{~nm} / \mathrm{H} \beta$ 

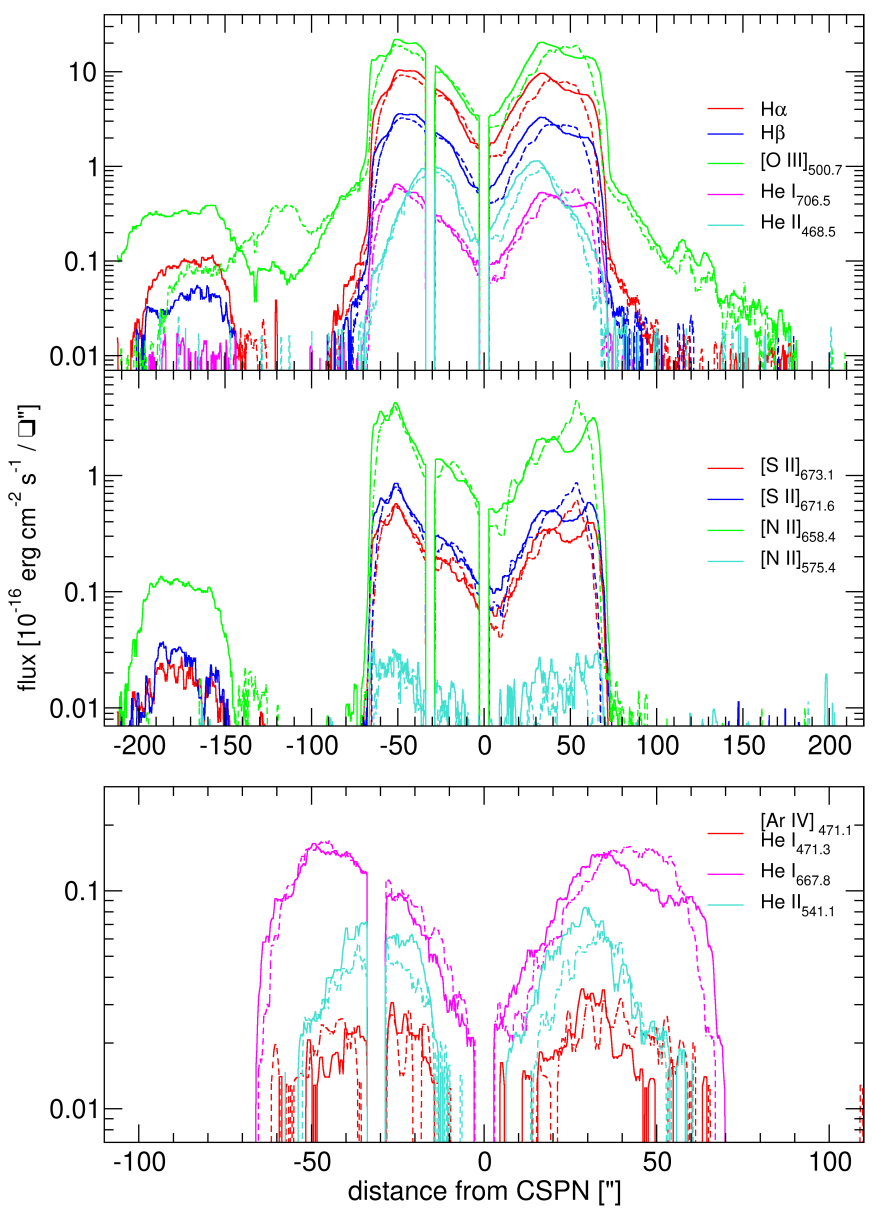

Fig. 8. Spatial distribution of the major spectral lines. Upper panel: brightest lines of oxygen, hydrogen and helium. Middle panel: the low ionized states of nitrogen and sulfur. The [N II] $654.8 \mathrm{~nm}$ line was skipped for clarity as it caries the same information as the [N II] $658.4 \mathrm{~nm}$ line. Lower panel: fainter lines of the two different ionization stages of helium. As the line around $471 \mathrm{~nm}$ shows the shape of the higher ionized He II lines, we have to assume that it is dominated by the [Ar IV] $471.1 \mathrm{~nm}$ emission and fewer contribution of the He I $471.3 \mathrm{~nm}$ emission. In all panels, solid lines denote to the slit at position angle $30^{\circ}$, while the dashed lines give the slit at $150^{\circ}$. The region contaminated by the central star was eliminated. Around $30^{\prime \prime}$ south of the nebula center, the FORS2 instrument has a chip gap.

appears constant at high values of seven to eight. On the other hand, the line ratio $R_{\mathrm{He}}=(\mathrm{He}$ II $468.6 \mathrm{~nm} / \mathrm{He}$ I $587.5 \mathrm{~nm})$ which is normally used to define for an excitation class of the nebula as a total (Ratag et al. 1997; Martins \& Viegas 2002), appears flat at $\approx 3.1$ for an inner radius of $30^{\prime \prime}$ and declines near perfectly linear to 0.5 out at $65^{\prime \prime}$. We derived the $\mathrm{H} \alpha / \mathrm{H} \beta$ ratio for both slit positions to test for a significant fraction or variations of the internal extinction within the nebula. Despite the differences in line intensities and some fluctuations between the two slit positions, we are unable to detect any of such variations (Fig. 9).

The $R_{\mathrm{N} \text { II }}=([\mathrm{N}$ II $] 654.8 \mathrm{~nm}+[\mathrm{N}$ II $] 658.4 \mathrm{~nm}) /[\mathrm{N}$ II $] 575.5 \mathrm{~nm}$ line ratio was used to derive an electron temperature as we shown in Fig. 10. The blue nitrogen line has a fairly low $\mathrm{S} / \mathrm{N}$, therefore the individual features observed at the line ratio should not be over-interpreted as intrinsic electron temperature variations, otherwise a spatial smoothing would be required. The temperatures lead to an average value of around $T_{\mathrm{e}}=11500 \mathrm{~K}$ using the calibrations given in Osterbrock \& Ferland (2006).

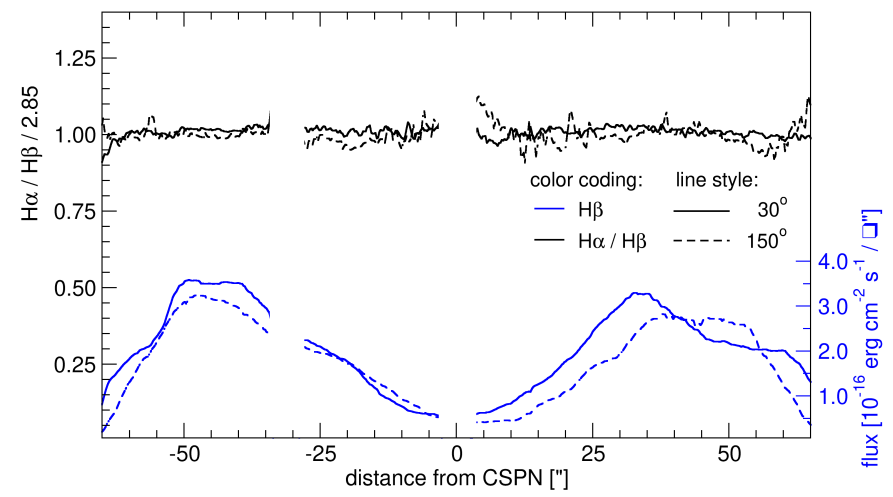

Fig. 9. $\mathrm{H} \alpha / \mathrm{H} \beta$ normalized to a Case $\mathrm{B}$ line ratio (Osterbrock \& Ferland 2006) to test for internal extinction.

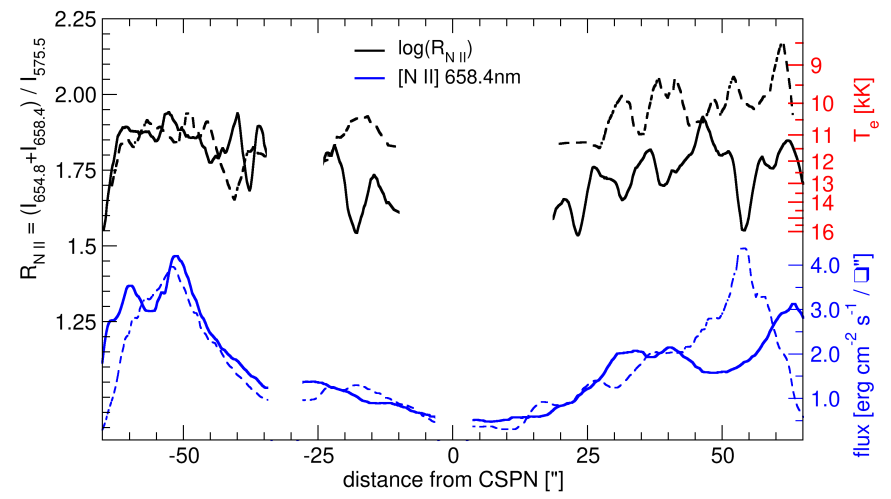

Fig. 10. Electron temperature derived from [N II] lines. Despite the large variation in intensity itself, the temperatures are fairly homogeneous. Line coding for the two slit directions is as in Fig. 9.

There is a slight tendency to asymmetry in the northern part of the nebula, but in general terms it appears homogeneous with an electron temperature well above $10^{4} \mathrm{~K}$. Unfortunately, there were no other temperature sensitive line triples like e.g. [O III], [Ar III] and [S III] covered in our spectral range. Temperatures derived from higher ionized states outside the colder clumps would have been of certain interest (see shock analysis Sect. 3.5).

Further we used the $R_{\mathrm{SII}}=([\mathrm{S}$ II $] 671.6 \mathrm{~nm} /[\mathrm{S}$ II $] 673.1 \mathrm{~nm})$ line ratio to derive the electron density $n_{\mathrm{e}}$ in the nebula. We used the average electron temperature $T_{\mathrm{e}}$ derived above and the two calibration $\rho\left(R_{\mathrm{S} I I}, T_{\mathrm{e}}\right)$ provided by Proxauf et al. (2014). The values are very constant at the lowest limit of the diagnostic diagram as shown in Fig. 11. Thus, the values must be interpreted mainly as upper limits. As the flux varies significantly and thus the integrated volume emissivity which is a function of the average density $\langle\rho\rangle$, we have to assume taking into account this nearly constant values for $\rho$, that the filling factor varies over the nebula. This was observed in the NIR molecular analysis for the nearby Helix nebula (Matsuura et al. 2009) and was also a result of the modeling of NGC 2438 in Öttl et al. (2014).

\subsection{Gas ionization tendency and shock heating}

The line ratios $\log (\mathrm{H} \alpha /[\mathrm{N} I \mathrm{II})$ versus $\log (\mathrm{H} \alpha /[\mathrm{S}$ II $])$ have been used in the past to identify photoionized $\mathrm{PNe}$ from that excited by additional mechanisms, such as found in supernova remnants or in H II regions. First introduced by Garcia Lario et al. (1991), 


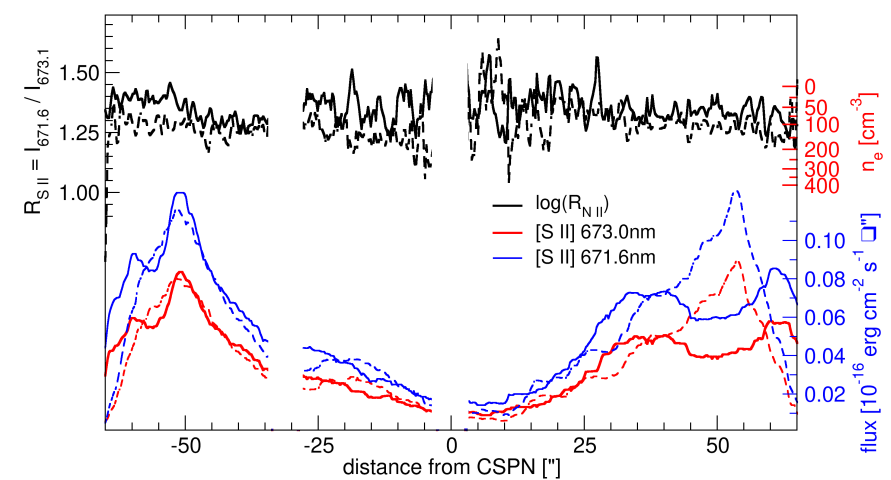

Fig. 11. Density sensitive line ratio of [S II] lines. Despite the large variation in intensity itself, the temperatures are fairly homogeneous. Line coding for the two slit directions is as in Fig. 9.

this scheme is refined in Magrini et al. (2003) and extensively reviewed by Frew \& Parker (2010). Öttl et al. (2014) extended this analysis to two dimensions to use it for the study of NGC 2438. We recently carried out a more detailed analysis using the multiple shell PNe NGC 3242, NGC 6826 and NGC 7662 (Barría \& Kimeswenger 2018). After investigating on their so called extended 2D diagnostic diagrams (E2DD) we concluded that major deviations from the inside to outside ionization tendency appeared only at regions were FLIERs or LISs are located. Previous studies on excitation properties of FLIERs and LISs (see e.g. Akras \& Gonçalves 2016; Akras et al. 2017), support for this scenario. Nevertheless, no FLIERs or LISs are visible in IC 5148, we followed the same strategy and studied the nebular ionization tendency through the main fragmentation structures in the nebula by means of its E2DD. As is shown in Fig. 12, where colors denote different nebular regions, we do not claim for regions at the main nebula showing significant deviations from the general ionization tendency. The regression line gives for a $1.218 \pm 0.016$ inclination. The fit does not include lines ratios from the halo structure. At the small PNe sample investigated in Barría \& Kimeswenger (2018) we noted a possible correlation between the slope of the fit in the E2DD and the CSPNe temperature. However, the previously studied nebula exhibit similar properties such as subsolar abundances, low expansion velocities, high excitation ranges, similar kinetic ages, and the presence of FLIERs/LISs. IC 5148, on the other hand, shows for a higher velocity expansion, no FLIERs/LISs, a solar abundance, and is highly evolved. Despite the fact that IC 5148 follow the tendency we found in the previously studied nebulae, this result has to be treated with caution, until a further study on the E2DD slope values considering a bigger and diversified sample of PNe could be conducted.

Following the ideas presented in Guerrero et al. (2013) to identify for shock heated regions, we derived the $[\mathrm{O}$ III]/H $\alpha$ in the FORS2 spectra as well as in the CDK20 images. As the seeing is a function of wavelength, we used the CSPN to derive a variation of the PSF. Considering the S/N on the central star was very high, we were able to identify not only the central component, but also the wide wings, known to happen as a result of rare scintillation events of the air. The combined PSF, giving for an error of far below 1\%, consists of a combined Gaussian with a component covering $94 \%$ of the peak intensity $(84 \%$ of the integrated flux) and a wing with a FWHM that is 3.5 times wider with $6 \%$ of the peak intensity ( $16 \%$ of the total flux). The width varied with wavelength as expected being about $7 \%$ larger

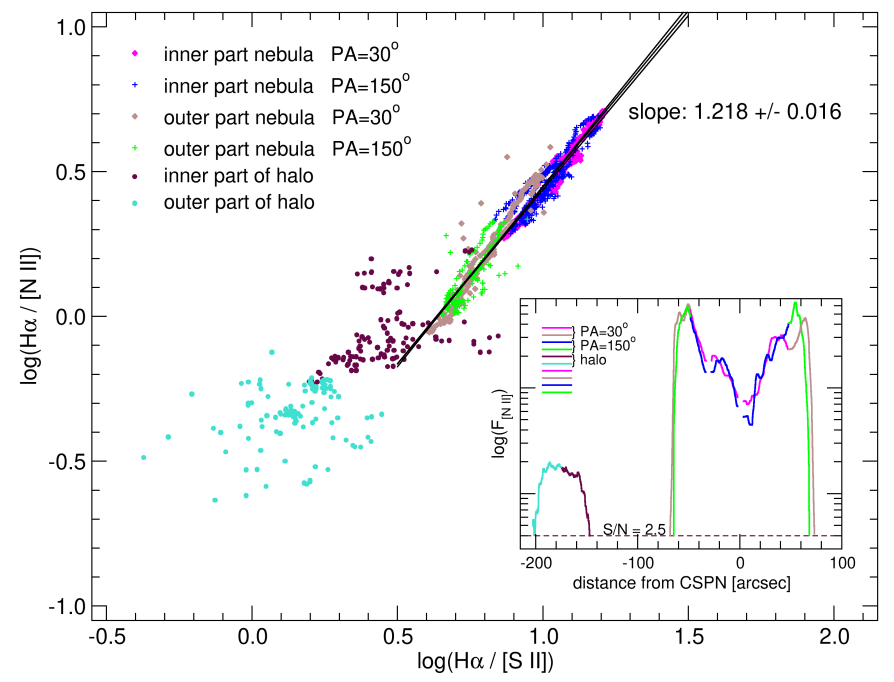

Fig. 12. Extended 2D diagnostic diagram of the nebula along the two slits positions and out to the halo in the regions with $S / N>3$ for all lines included in the graph.

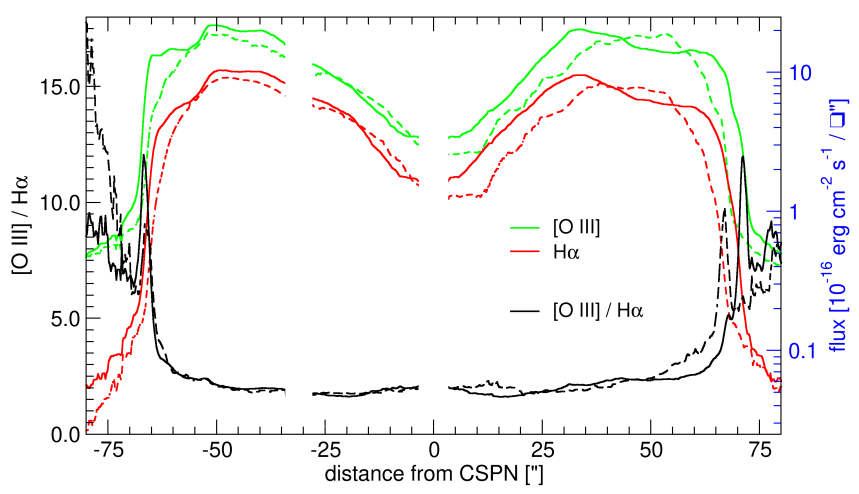

Fig. 13. Shock front analysis along the slit in the two FORS2 spectra using $\leq[\mathrm{O} I I I] / \mathrm{H} \alpha$. The spectra were folded with a PSF derived from the central star to obtain same resolution. Solid and dashed lines indicate for the $30^{\circ}$ and $150^{\circ}$ slit spectra, respectively.

at $500 \mathrm{~nm}\left(1^{\prime \prime} .05\right)$ than that we found at $660 \mathrm{~nm}(0.98)$. Nevertheless, the wide wing reaches out to 3 .' 0 , containing a significant contribution to the total flux. This is of special importance as the line flux drops in the region of interest by more than a order of magnitude. While the main nebula shows a very homogeneous line ratio of around 2.5 (see Fig. 13), both, the latter spectral analysis as well as the image analysis displayed at Fig. 14, claim for shock features revealed by a thin shell of enhanced oxygen emission with line ratios significantly higher than ten. According to Guerrero et al. (2013), this skin of shock-excited material develops when a fast shell expands into a lower density medium. Moreover, here also the Mach number changes. To test the reliability of this result, the data were also folded with two and three times the derived PSF. As the feature in the line ratio gets only slightly weaker, respectively, by 20 and $60 \%$ in the ratio, but still giving a line ratio of five, we can be sure that it is not an artifact of the data analysis. Moreover, we find a slight steady increase already in the last $8-12^{\prime \prime}$ inside the front, where the intensity itself resembles still a nearly constantly high flux. We assume that this is caused by the afterglow of the heating in the postshock region. The $\mathrm{S} / \mathrm{N}$ of $\mathrm{H} \alpha$ images as well as for the spectra 


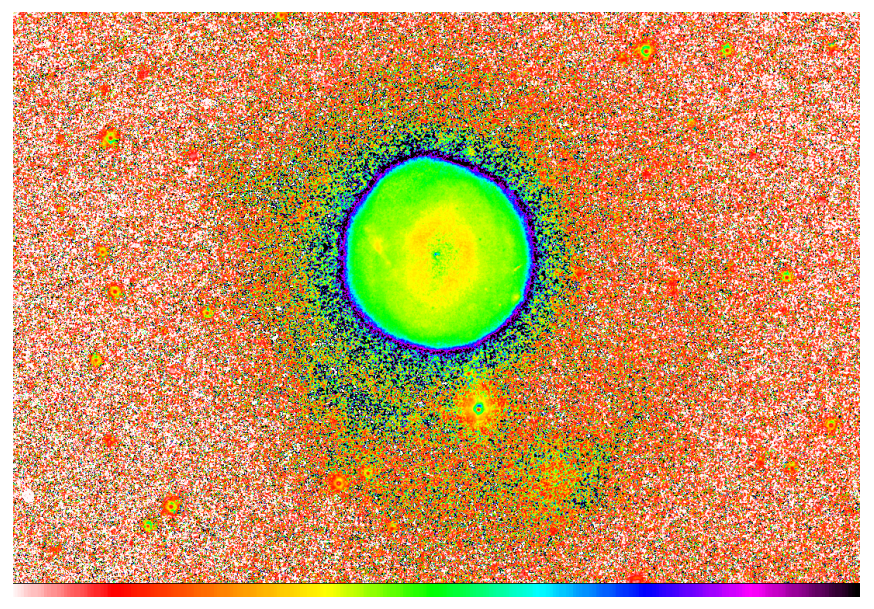

Fig. 14. Shock front analysis as in Fig. 13 but using the CDK20 narrow band images. The color coding is linear for $0 \leq[\mathrm{O}$ III $] / \mathrm{H} \alpha \leq 5$.

in the halo is not sufficient to derive reasonable numbers for the regions outside the main nebula.

\subsection{Cloudy model}

Physical parameters of the different regions along the main nebula, were investigated by means of Cloudy modeling. The model was performed with version 17.00 of Cloudy (Ferland et al. 2017) and covering only the main nebula. The Cloudy code makes use of an extensive atomic and molecular database together to the possibility to include grains and incorporates a filling factor to simulate clumpiness.

The previously derived CSPN parameters such as the effective temperature, luminosity and surface gravity listed in Table 1, were used in the model as input parameters for the ionizing source. Thus, we modeled for a central initial mass star of $M_{\text {ini }}=1.5 M_{\odot}$, a metallicity of $Z=0.02$ dex and a modeled age of $8500 \mathrm{yr}$. The latter measured from the point where the postAGB object has a $\log \left(T_{\text {eff }}\right)=3.85$ (Miller Bertolami 2016). The spectral energy distribution of the CSPN was obtained via stateof-the-art H-Ni NLTE model stellar atmospheres provided by Rauch (2003). An expanding spherical geometry was assumed for the nebula. The net line emission provided by Cloudy per volume-unit ( $\mathrm{erg} \mathrm{cm}^{-3} \mathrm{~s}^{-1}$ ), was used to create a model 2D projected emissivity profile along the line of sight by an in-house IDL code. In this way, we were able to make a direct comparison of the observed emissivity profiles to the modeled ones. Abundances were set in the Cloudy model to that of a typical metal rich PN as incorporated in the model code from Aller \& Czyzak (1983) and Khromov (1989). An assumed Gaia DR2 distance of $1.3 \mathrm{kpc}$ was used. Although, we also attempt to model the nebula by using the two additional intersection distance solutions found in Sect. 3.3 (1.8 and $1.5 \mathrm{kpc}$ ). However, the use of these higher distances did not reach convergence for a good model.

The modeling was carried out setting an initial tabulated density profile derived from the $\mathrm{H} \alpha$ emissivity (see details of this procedure in Barría \& Kimeswenger 2018). As was pointed out in Sect. 3.4, despite the large intensity variation of the [S II] lines, the density sensitive line ratio $R_{\mathrm{SII}}$ is fairly constant. Hence, we have to assume that the filling factor varies through the nebula. Variations in clumpiness and filling factors were also observed in NGC 2438 in the same way (Öttl et al. 2014) and resolved even in molecular lines in other nearby nebulae (e.g. Matsuura et al. 2009 , and references therein). The estimated line ratio $R_{\text {SII }} \sim 1.4$

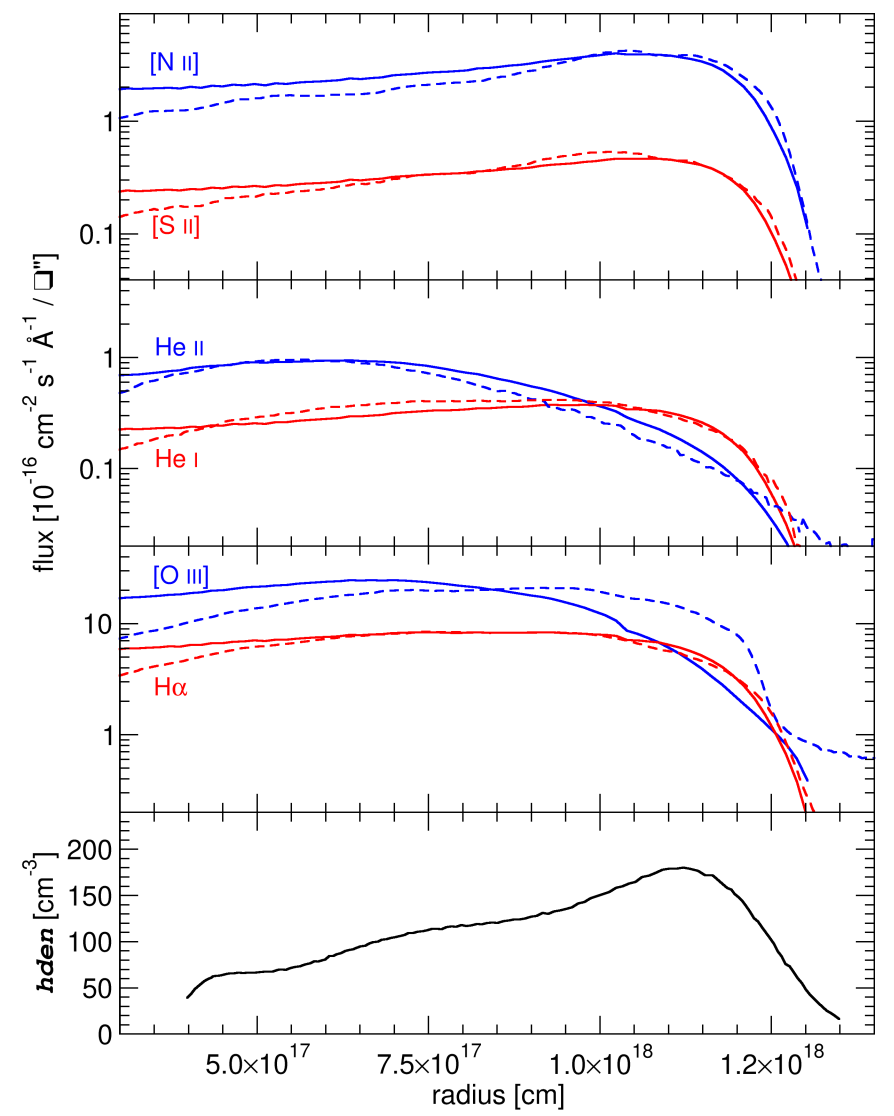

Fig. 15. Best fit Cloudy model. Solid lines are giving the model, dashed lines the data averaged in the four directions taken in the slit. Lowest panel: derived Cloudy hden density profile.

(see Sect. 3.4) give us an upper limit for the electron density of $n_{\mathrm{e}} \sim 200 \mathrm{~cm}^{-3}$ (Osterbrock \& Ferland 2006; Proxauf et al. 2014). It has to be noted here, that the hydrogen density profile in Cloudy is representative for the density within the filled fraction of the nebulosity. In this sense, the integrated nebular mass for such a clumpy model can only be achieved by multiplying the density by the corresponding filling factor in each region. Based on this initial density profile as a starting point, we manually iterated to convergence for the final model using the observed line profiles of [N II] $658.3 \mathrm{~nm},\left[\mathrm{~S}_{\mathrm{II}}\right] 673.0 \mathrm{~nm}, \mathrm{He}$ I $587.5 \mathrm{~nm}$, $\mathrm{He}$ II $468.5 \mathrm{~nm}$, [O III] $500.7 \mathrm{~nm}$ and $\mathrm{H} \alpha 656.3 \mathrm{~nm}$. The observed profiles were obtained by averaging the four probes taken radially by the two slit directions. Convergence was derived only by dividing the main nebula into three independent spherical shell regions with independently filling factors as free parameters. The final model was achieved after added the resulting best models for each region (see Fig. 15). Final derived Cloudy density profile (called hden) is also shown in Fig. 15. The resulting parameters for the best nebula model are listed in Table 2.

While we notice very good agreement between the observed and modeled [N II], [S II], He I, He II and $\mathrm{H} \alpha$ emissivity profiles, we observed moderate deviations in the [O III] profile. A modeled underestimation on the [O III] emission becomes more evident at the outer regions of the main nebula. Considering this, we suggest here for two possible scenarios: one involving emission from a thin hot interclump medium and, a second one related to the residual heating of the post-shock afterglow. The Cloudy code definition of the filling factor assumes that the mass is distributed solely in the clumps. Thus, a possible 
Table 2. Main derived nebular properties and nebular parameters obtained from the best Cloudy model.

\begin{tabular}{ll}
\hline \hline Nebular parameter & Value \\
\hline Expansion velocity & $47.9 \pm 1.5\left(\mathrm{~km} \mathrm{~s}^{-1}\right)$ \\
Model age & $8500 \mathrm{yr}$ \\
Average $T_{\mathrm{e}}$ & $11500(\mathrm{~K})$ \\
Main nebula angular radius & $68^{\prime \prime}$ \\
Main nebula linear radius & $1.32 \times 10^{18}(\mathrm{~cm})=0.43(\mathrm{pc})$ \\
Total H mass & $0.34\left(M_{\odot}\right)$ \\
Region I & $(3.98-4.70) \times 10^{17}(\mathrm{~cm})$ \\
Radius & 0.18 \\
Filling factor & $0.0015 M_{\odot}$ \\
H mass & \\
Region II & $(0.47-1.04) \times 10^{18}(\mathrm{~cm})$ \\
Radius & 0.37 \\
Filling factor & $0.15 M_{\odot}$ \\
H mass & \\
Region III & $(1.04-1.32) \times 10^{18}(\mathrm{~cm})$ \\
Radius & 0.29 \\
Filling factor & $0.18\left(M_{\odot}\right)$ \\
Hydrogen mass & \\
\hline
\end{tabular}

Notes. The independent model regions are labeled from in to out as Regions I, II, and III.

additional contribution from ionized [O III] gas coming from the interclump medium, cannot be considered for our Cloudy clumpy model. Under the second proposed scenario, spectroscopic and image analysis gives evidence for a thin layer of enhanced emission due to a shock front. We might consider then for a possible enhancement of the [O III] emission, as a result of the heated post-shock region. This corresponds well also to the previous line analysis shown in Fig. 13.

\section{Nebular halo}

A very special set of features in this nebula is occupied by the outer halo. While many nebulae in this evolutionary stage do show one or even more halos, this one has a few very special features to be mentioned in detail.

\subsection{Inner halo}

The inner halo is well represented by an exponential decline of the intensity into nearly all four slit directions equally (Fig. 16). It can be detected in $\mathrm{H} \alpha$ and [N II] $(658.4 \mathrm{~nm})$ up to a radius of about $100^{\prime \prime}$ (corresponding to $0.85 \mathrm{pc}$ at a distance of $1.3 \mathrm{kpc}$ ) with a constant line ratio of $\mathrm{H} \alpha /[\mathrm{N} \mathrm{II}](658.4 \mathrm{~nm}) \approx 4$. A similar (or only marginally flatter) decline is seen for the [O III] line, which is nearly an order of magnitude brighter. Hence, this one can be followed up to the very outskirts (Fig. 16). All four slit positions give about the same [O III] decline until a radius of $110^{\prime \prime}$. Beyond that, only the two northern ones, not influenced by the outer halo bow (see Sect. 4.3), seem to continue straight as before and after the halo rings (between 110 and 135"; see Sect. 4.2). At $175^{\prime \prime}$ (corresponding to $1.5 \mathrm{pc}$ at $D=1.3 \mathrm{kpc}$ ) both signals vanishes in the noise. The high $[\mathrm{O}$ III $] / \mathrm{H} \alpha \approx 8$ (even with a slight steady increase toward larger radius) implies that the halo is ionized to a high degree (see modeling and discussion of the halo in NGC 2438 by Öttl et al. 2014). This ratio and its steady increase is caused by the hardening of the radiation due to the rapid decline of the absorption efficiency of hydrogen

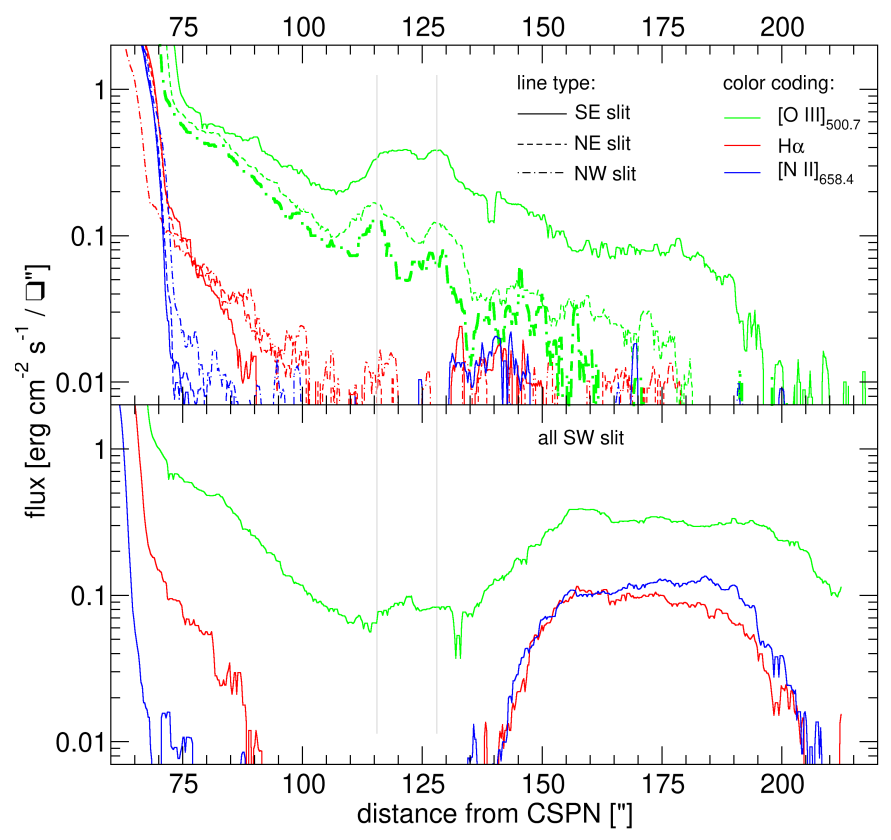

Fig. 16. Brightest emission lines in the halo. Upper panel: two northern and one southeast section of the halo. Lower panel: southwest section. The location of the two halo rings are marked by gray lines.

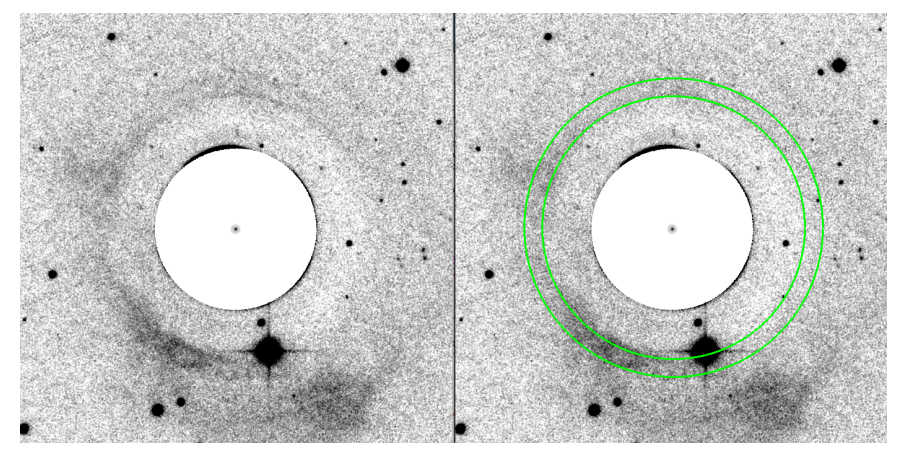

Fig. 17. [O III] image of the halo region. Left panel: after masking the main nebula and removing a Gaussian shaped decline representing the normal halo intensity distribution. Right panel: same image with two circles centered exactly at the CSPN at 115 and $128^{\prime \prime}$.

$\kappa_{H} \propto \chi^{-3}$ for photon energies $\chi>1$ Ryd (Preite-Martinez \& Pottasch 1983). The effect for the halos of MSPNe is discussed in detail in Guerrero \& Manchado (1999). As we cannot measure the density of the halo directly, a low density $\rho \propto r^{-2}$ was used to test the continuum radiation transmitted by the CLOUDY model. The incident radiation to the halo is depleted between 1.1 and 2.5 Ryd to more than $85 \%$, while the very hot CSPN having its radiation maximum above 3 Ryd is still very strong at ionization energies of He II and O III. The result fit well to this assumption of a photoionized material. As we do not have decent density information tracers, the results can used only to follow the tendency and not can be used in absolute quantities.

\subsection{Halo rings}

Multiple outer faint rings and arcs have been found at several planetary nebulae among different morphological types (see e.g. Terzian \& Hajian 2000; Corradi et al. 2004; Ramos-Larios et al. 2016). While several different formation mechanisms have been 
proposed to explain these structures, their origin is still not that clear, but strongly connected to the mass-loss processes at the latest phase of the AGB. While these features appears scarcely among roundish $\mathrm{PNe}$ ( 7\%, Ramos-Larios et al. 2016), we found in the [O III] images of IC 5148, at a distance of 115 and 128", corresponding to 1.00 and $1.08 \mathrm{pc}$ at $D=1.3 \mathrm{kpc}$, respectively, two exact concentric rings centered perfectly respect to the central star. Moreover, the images are deep enough to exclude the typically found multiple structures down by nearly an order of magnitude in brightness. To enhance visibility a power law shaped decline of the regular halo was subtracted from the images (see Fig. 17). The striking is not only the perfect circular shape, but also the fact, that they increase the $[\mathrm{O}$ III $] / \mathrm{H} \alpha$ ratio to at least 30 (lower limit), as these rings are invisible in the other lines. According to Corradi et al. (2004), the spacing between rings correlates with the post-AGB age of the nebula. Although IC 5148 appears older than the nebulae sample studied by these authors, the large spacing between the rings found here ( $\Delta \theta=13^{\prime \prime}, \Delta r=0.08 \mathrm{pc}$ at $1.3 \mathrm{kpc}$ ) reinforces the likelihood of this being a quite-evolved nebula.

To date, the most plausible scenario for the formation of such structures is correlated to periodic or quasi-periodic enhancements in the mass-loss rate at the end of the AGB phase. As Ramos-Larios et al. (2016) reviewed, several mechanisms have been suggested as being responsible for these periodic or quasiperiodic fluctuations such as solar-like magnetic activity in AGB stars (Soker 2000), long-period oscillations in AGB stars (Icke et al. 1992), viscous momentum transfer between grains and gas (Simis et al. 2001) or even binary interactions (Kim \& Taam 2012a,b). Some other mechanisms as thermal pulses or AGB pulsations have been dismissed basically due to an inconsistency of the time lapses between successive observed rings. But these scenarios produce normally either only a single (or widely spaced) rings or a large number of them. Especially the dustbased mechanisms (e.g., Simis et al. 2001) are dominated by reflected light of the main nebula, which would not change the line ratio so much. Certainly a deep study on the origin of the rings found in IC 5148 is far beyond the scope of this paper, however as an alternative scenario and following the discussion about shock signatures in Guerrero et al. (2013), we proposed that these structures might be caused by shock fronts. As the other lines, especially also [S II] are not detected anymore out there, these features require deeper observations for further verification.

\subsection{Outer halo bow}

The "bow" is in the outermost part of the halo, peaking into a diffuse clump in the SW region and having a decent visible tail until the SE slit position. It extends from 145 to $200^{\prime \prime}$ from the central star, which corresponds to $0.92-1.26 \mathrm{pc}$ at a distance of $1.3 \mathrm{kpc}$. It is at a completely different excitation state than the ring features described above. As shown in Fig. 16 it has an $\mathrm{H} \alpha /[\mathrm{N} \mathrm{II}]$ $(658.4 \mathrm{~nm})$ line ratio around unity and [O III] $(500.7 \mathrm{~nm}) / \mathrm{H} \alpha$ of about 3 . In this respect, it almost exactly resembles the state of the central nebula and gives an excitation below that found in the innermost halo region (from 70 to $100^{\prime \prime}$ ) inside the rings. It nicely continues the excitation direction of the main nebula from inside to outside as shown in Fig. 12. Thus, it is most likely photoionized material. Weather it was an unusual asymmetric mass loss event on the AGB, or interaction with ISM remains unclear. Although the latter is fairly unlikely at this large distance from the galactic plane. Also, the low foreground extinction and the homogeneity of the Balmer line ratio over the whole nebula indicate that a patchy cloudy environment is very unlikely.

\section{Conclusions}

We performed a detailed photometric and spectroscopic analysis of the high galactic latitude roundish nebula IC 5148, an older evolved nebulae, undisturbed from interaction with the interstellar matter. We find that the distance derived from Gaia DR2 corresponds well to those obtained by statistical methods. Moreover, the very blue color in the Gaia photometry indicates, as shown in Kimeswenger \& Barría (2018), a good separation of the star from the nebula and give us confidence in the low formal error given by the trigonometric solution of Gaia which results in a distance of $D=1.28_{-0.13}^{+0.16} \mathrm{kpc}$. Furthermore, we were able to derive a more reliable interstellar foreground extinction leading to $E(B-V)=0$. 03 with different independent methods. This is significantly lower than the hitherto often-used values of Kaler et al. (1990) and Kingsburgh \& Barlow (1994). Mediumhigh resolution spectroscopy was used to derive a central star temperature of $140_{13}^{+5} \mathrm{kK}$. However, after comparison to state-ofthe-art evolutionary tracks (Miller Bertolami 2016) we obtain a real value to be slightly lower and thus we adopted $130 \mathrm{kK}$ and a gravity of $\log (g) \approx 7$ on the white dwarf cooling track. The progenitor therefore must have been a low mass star of initially $1.5 M_{\odot}$. This fits well to the expectation that very massive stars should not be found so far from the galactic plane. We find from our observations of the central star, as well as from the nebular investigation, that IC 5148, despite its high galactic $z$, is not, similar to other nebulae in these regions (e.g. A 15, A 20, MeWe 1-3; Emprechtinger et al. 2004), underabundant in metals. This was somewhat unexpected and in contradiction to the postulation of Soker (2002), that spherical PNe might form only in metal poor environment.

IC 5148 shows a perfect shock structure at its outermost edge of the main nebula, as predicted in the hydrodynamic models (e.g. Perinotto et al. 2004). It is only visible by using the high ionized species as already suggested and discussed in Guerrero et al. (2013). The shock sensitive diagrams, using low ionized species following Magrini et al. (2003), are not sensitive to this effect in the outermost regions.

The presented CLOUDY photoionization model shows generally a self-consistent model of the nebula. However, the observed excess of the forbidden [O III] line could not be modeled in that way. We speculate that this might be an afterglow of the after-shock region, responsible for driving up the less frequent oxygen atoms even by some small rest of overheated helium. A dedicated time-dependent radiative transfer code with source terms of shock heating and afterglows would be required for such a model.

The reported discovery of structured halo emission is enigmatic. The inner halo region resembles the perfect circular shape as that of the main nebula, while further out a bow like structure is seen at one side of the nebula only. While the latter shows line ratios as seen from normal photoionized material, the spherical rings have an extremely large $[\mathrm{O}$ III] $/ \mathrm{H} \alpha$. Spectroscopy even deeper than ours would be required to resolve this puzzle.

Acknowledgements. We would like to thank the referee Martin Guerrero for his helpful comments. The study is based on observations made with ESO Telescopes at the La Silla Paranal Observatory under program ID 098.D-0332. This work has made use of data from the European Space Agency (ESA) mission Gaia (https: //www. cosmos.esa.int/gaia), processed by the Gaia Data Processing and Analysis Consortium (DPAC, https://www.cosmos.esa.int/web/ gaia/dpac/consortium). Furtheron this research has made use of the SIMBAD database, operated at CDS, Strasbourg, France (Wenger et al. 2000), the NASA/IPAC Extragalactic Database (NED) which is operated by the Jet Propulsion Laboratory, California Institute of Technology, under contract with the National Aeronautics and Space Administration and has made use of "Aladin Sky 
Atlas" developed at CDS, Strasbourg Observatory, France (Bonnarel et al. 2000). D.B. and this research were financed by the FONDO ALMA-Conicyt Programa de Astronomía/PCI 31150001 and W.K. is funded by the Hochschulraumstrukturmittel provided by the Austrian Federal Ministry of Education, Science and Research (BMBWF).

\section{References}

Acker, A., Gleizes, F., Chopinet, M., et al. 1982, Publication Speciale du Centre de Donnees Stellaires, 3

Acker, A., Marcout, J., Ochsenbein, F., et al. 1992, The Strasbourg-ESO Catalogue of Galactic Planetary Nebulae. Parts I, II. (European Southern Observatory)

Akras, S., \& Gonçalves, D. R. 2016, MNRAS, 455, 930

Akras, S., Gonçalves, D. R., \& Ramos-Larios, G. 2017, MNRAS, 465, 1289

Aller, L. H., \& Czyzak, S. J. 1983, ApJS, 51, 211

Appenzeller, I., Fricke, K., Fürtig, W., et al. 1998, The Messenger, 94, 1

Balick, B. 1987, AJ, 94, 671

Barría, D., \& Kimeswenger, S. 2018, MNRAS, 480, 1626

Bessell, M. S. 1990, PASP, 102, 1181

Bessell, M. S., Castelli, F., \& Plez, B. 1998, A\&A, 333, 231

Blöcker, T. 1995, A\&A, 299, 755

Bojičić, I. S., Parker, Q. A., \& Frew, D. J. 2017, in Planetary Nebulae: Multi-Wavelength Probes of Stellar and Galactic Evolution, eds. X. Liu L. Stanghellini, \& A. Karakas, IAU Symp., 323, 327

Bonnarel, F., Fernique, P., Bienaymé, O., et al. 2000, A\&AS, 143, 33

Buckley, D., \& Schneider, S. E. 1995, ApJ, 446, 279

Cahn, J. H., Kaler, J. B., \& Stanghellini, L. 1992, A\&AS, 94, 399

Chu, Y.-H., Jacoby, G. H., \& Arendt, R. 1987, ApJS, 64, 529

Corradi, R. L. M., Schönberner, D., Steffen, M., \& Perinotto, M. 2000, A\&A, 354,1071

Corradi, R. L. M., Sánchez-Blázquez, P., Mellema, G., Gianmanco, C., \& Schwarz, H. E. 2004, A\&A, 417, 637

Dreyer, J. L. E. 1910, MNRAS, 59, 105

Emprechtinger, M., Forveille, T., \& Kimeswenger, S. 2004, A\&A, 423, 1017

Emprechtinger, M., Rauch, T., \& Kimeswenger, S. 2005, A\&A, 431, 215

Ferland, G. J. 2000, Rev. Mex. Astron. Astrofis., 27, 153

Ferland, G. J., Korista, K. T., Verner, D. A., et al. 1998, PASP, 110, 761

Ferland, G. J., Porter, R. L., van Hoof, P. A. M., et al. 2013, Rev. Mex. Astron. Astrofis, 49, 137

Ferland, G. J., Chatzikos, M., Guzmán, F., et al. 2017, Rev. Mex. Astron. Astrofis., 53, 385

Frank, A., \& Mellema, G. 1994, A\&A, 289, 937

Freudling, W., Romaniello, M., Bramich, D. M., et al. 2013, A\&A, 559, A96

Frew, D. J., \& Parker, Q. A. 2010, PASA, 27, 129

Frew, D. J., Parker, Q. A., \& Bojičić I. S. 2016, MNRAS, 455, 1459

Gaia Collaboration (Brown, A. G. A., et al.) 2018, A\&A, 616, A1

Gale, W. F. 1897, Astron. Nachr., 143, 293

Garcia Lario, P., Manchado, A., Riera, A., Mampaso, A., \& Pottasch, S. R. 1991, A\&A, 249, 223

Gesicki, K., Zijlstra, A. A., Hajduk, M., \& Szyszka, C. 2014, A\&A, 566, A48

Guerrero, M. A., \& Manchado, A. 1999, ApJ, 522, 378

Guerrero, M. A., Toalá, J. A., Medina, J. J., et al. 2013, A\&A, 557, A121

Hoffmeister, C. 1961, Die Sterne, 37, 204

Hua, C. T., Dopita, M. A., \& Martinis, J. 1998, A\&AS, 133, 361

Icke, V., Frank, A., \& Heske, A. 1992, A\&A, 258, 341

Kaler, J. B., \& Feibelman, W. A. 1985, ApJ, 297, 724

Kaler, J. B., Shaw, R. A., \& Kwitter, K. B. 1990, ApJ, 359, 392

Kausch, W., Noll, S., Smette, A., et al. 2015, A\&A, 576, A78

Khromov, G. S. 1989, Space Sci. Rev., 51, 339

Kim, H., \& Taam, R. E. 2012a, ApJ, 744, 136

Kim, H., \& Taam, R. E. 2012b, ApJ, 759, 59

Kimeswenger, S., \& Barría, D. 2018, A\&A, 616, L2

Kingsburgh, R. L., \& Barlow, M. J. 1994, MNRAS, 271, 257

Kwok, S. 1982, ApJ, 258, 280
Kwok, S. 2002, in Interacting Winds from Massive Stars, eds. A. F. J. Moffat \& N. St-Louis, ASP Conf. Ser., 260, 245

Kwok, S., Purton, C. R., \& Fitzgerald, P. M. 1978, ApJ, 219, L125

Landolt, A. U. 1992, AJ, 104, 340

Landolt, A. U., \& Uomoto, A. K. 2007, AJ, 133, 768

Lindegren, L., Hernández, J., Bombrun, A., et al. 2018, A\&A, 616, A2

Luri, X., Brown, A. G. A., Sarro, L. M., et al. 2018, A\&A, 616, A9

Magrini, L., Perinotto, M., Corradi, R. L. M., \& Mampaso, A. 2003, A\&A, 400, 511

Martins, L. P., \& Viegas, S. M. 2002, A\&A, 387, 1074

Matsuura, M., Speck, A. K., McHunu, B. M., et al. 2009, ApJ, 700, 1067

Meatheringham, S. J., Wood, P. R., \& Faulkner, D. J. 1988, ApJ, 334, 862

Mellema, G., \& Frank, A. 1995, MNRAS, 273, 401

Mendez, R. H. 1991, Evolution of Stars: the Photospheric Abundance Connection (the Netherlands: Springer), 145, 375

Miller Bertolami M. M. 2016, A\&A, 588, A25

Modigliani, A., Goldoni, P., Royer, F., et al. 2010, in Observatory Operations: Strategies, Processes, and Systems III, Proc. SPIE, 7737, 773728

Napiwotzki, R. 1999, A\&A, 350, 101

Napiwotzki, R. 2001, A\&A, 367, 973

Noll, S., Kausch, W., Kimeswenger, S., et al. 2014, A\&A, 567, A25

Okorokov, V. A., Shustov, B. M., Tutukov, A. V., \& Yorke, H. W. 1985, A\&A, 142,441

Osterbrock, D. E., \& Ferland, G. J. 2006, Astrophysics of Gaseous Nebulae and Active Galactic Nuclei (Sausalito, CA: University Science Books)

Öttl, S., Kimeswenger, S., \& Zijlstra, A. A. 2014, A\&A, 565, A87

Parker, Q. A., Bojičić, I. S., \& Frew, D. J. 2016, J. Phys. Conf. Ser., 728, 032008

Patriarchi, P., \& Perinotto, M. 1991a, A\&AS, 91, 325

Patriarchi, P., \& Perinotto, M. 1991b, Proc. Astron. Soc. Aust., 9, 309

Perinotto, M., Kifonidis, K., Schoenberner, D., \& Marten, H. 1998, A\&A, 332, 1044

Perinotto, M., Schönberner, D., Steffen, M., \& Calonaci, C. 2004, A\&A, 414, 993

Phillips, J. P. 2005, MNRAS, 357, 619

Preite-Martinez, A., \& Pottasch, S. R. 1983, A\&A, 126, 31

Proxauf, B., Öttl, S., \& Kimeswenger, S. 2014, A\&A, 561, A10

Ramos-Larios, G., Santamaría, E., Guerrero, M. A., et al. 2016, MNRAS, 462, 610

Ratag, M. A., Pottasch, S. R., Dennefeld, M., \& Menzies, J. 1997, A\&AS, 126, 297

Rauch, T. 2003, A\&A, 403, 709

Rauch, T., \& Ringat, E. 2011, Astronomical Data Analysis Software and Systems XX, eds. I. N. Evans, A. Accomazzi, D. J. Mink, \& A. H. Rots, ASP Conf. Ser., 442, 563

Rauch, T., \& Reindl, N. 2014, Asymmetrical Planetary Nebulae VI Conf., 77

Schlafly, E. F., \& Finkbeiner, D. P. 2011, ApJ, 737, 103

Schönberner, D., Jacob, R., Lehmann, H., et al. 2014, Astron. Nachr., 335, 378

Simis, Y. J. W., Icke, V., \& Dominik, C. 2001, A\&A, 371, 205

Smette, A., Sana, H., Noll, S., et al. 2015, A\&A, 576, A77

Soker, N. 2000, ApJ, 540, 436

Soker, N. 2002, A\&A, 386, 885

Stanghellini, L., Shaw, R. A., \& Villaver, E. 2008, ApJ, 689, 194

Stanghellini, L., Bucciarelli, B., Lattanzi, M. G., \& Morbidelli, R. 2017, New A, 57,6

Steffen, M., Szczerba, R., \& Schoenberner, D. 1998, A\&A, 337, 149

Swift, L. 1899, MNRAS, 59, 568

Tajitsu, A., \& Tamura, S. 1998, AJ, 115, 1989

Terzian, Y., \& Hajian, A. R. 2000, in Asymmetrical Planetary Nebulae II: From Origins to Microstructures, eds. J. H. Kastner, N. Soker, \& S. Rappaport, ASP Conf. Ser., 199, 33

van de Steene, G. C., \& Zijlstra, A. A. 1994, A\&AS, 108, 485

Vassiliadis, E., \& Wood, P. R. 1993, ApJ, 413, 641

Vassiliadis, E., \& Wood, P. R. 1994, ApJS, 92, 125

Vernet, J., Dekker, H., D’Odorico, S., et al. 2011, A\&A, 536, A105

Weinberger, R. 1989, A\&AS, 78, 301

Wenger, M., Ochsenbein, F., Egret, D., et al. 2000, A\&AS, 143, 9

Zhang, C. Y. 1995, ApJS, 98, 659 